\title{
Numerical solution of open string field theory in Schnabl gauge
}

\section{E. Aldo Arroyo, A. Fernandes-Silva and R. Szitas}

Centro de Ciências Naturais e Humanas, Universidade Federal do ABC, Santo André, 09210-170 São Paulo, SP, Brazil

E-mail: aldo.arroyo@ufabc.edu.br, armando.silva@aluno.ufabc.edu.br, renato.szitas@aluno.ufabc.edu.br

ABSTRACT: Using traditional Virasoro $L_{0}$ level-truncation computations, we evaluate the open bosonic string field theory action up to level $(10,30)$. Extremizing this level-truncated potential, we construct a numerical solution for tachyon condensation in Schnabl gauge. We find that the energy associated to the numerical solution overshoots the expected value -1 at level $L=6$. Extrapolating the level-truncation data for $L \leq 10$ to estimate the vacuum energies for $L>10$, we predict that the energy reaches a minimum value at $L \sim 12$, and then turns back to approach -1 asymptotically as $L \rightarrow \infty$. Furthermore, we analyze the tachyon vacuum expectation value (vev), for which by extrapolating its corresponding level-truncation data, we predict that the tachyon vev reaches a minimum value at $L \sim 26$, and then turns back to approach the expected analytical result as $L \rightarrow \infty$.

Keywords: String Field Theory, Tachyon Condensation

ARXIV EPRINT: 1707.09452 


\section{Contents}

1 Introduction 1

2 Level truncation and Schnabl gauge $\quad 4$

3 Effective tachyon potential and its branch structure $\quad 9$

3.1 Branch structure in Siegel gauge 9

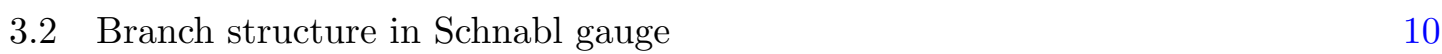

4 Extrapolation techniques and the vacuum energy analysis 13

$\begin{array}{lll}4.1 & \text { Gaiotto-Rastelli extrapolation technique } & 14\end{array}$

$\begin{array}{lll}\text { 4.1.1 Siegel gauge } & 14\end{array}$

$\begin{array}{lll}\text { 4.1.2 Schnabl gauge } & 15\end{array}$

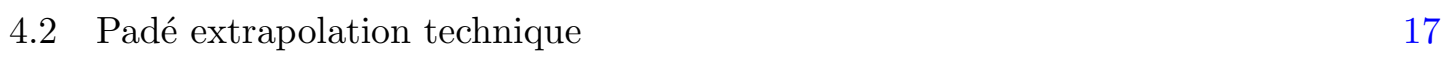

$\begin{array}{lll}\text { 4.2.1 Siegel gauge } & 18\end{array}$

$\begin{array}{lll}4.2 .2 & \text { Schnabl gauge } & 20\end{array}$

5 The tachyon vev 21

5.1 Siegel gauge 22

$\begin{array}{ll}5.2 & \text { Schnabl gauge } 25\end{array}$

$\begin{array}{lll}6 & \text { Summary and discussion } & 28\end{array}$

\section{Introduction}

Schnabl's solution for tachyon condensation [1] in Witten's open bosonic string field theory [2] has been a remarkable achievement which has provided an elegant analytic proof of Sen's first conjecture [3, 4]. Schnabl's seminal work has allowed the development of modern analytical and numerical techniques [5-21] which have been used to explore new analytic solutions [22-33], and the bosonic results have been extended to the case of open superstring field theories [34-45].

There are two ways of writing Schnabl's analytic solution; the first way is in terms of the Bernoulli numbers $B_{n}[1,7,23]$,

$$
\begin{aligned}
\Psi & =\sum_{n, p} f_{n, p}\left(\mathcal{L}_{0}+\mathcal{L}_{0}^{\dagger}\right)^{n} \tilde{c}_{p}|0\rangle+\sum_{n, p, q} f_{n, p, q}\left(\mathcal{B}_{0}+\mathcal{B}_{0}^{\dagger}\right)\left(\mathcal{L}_{0}+\mathcal{L}_{0}^{\dagger}\right)^{n} \tilde{c}_{p} \tilde{c}_{q}|0\rangle, \\
f_{n, p} & =\frac{1-(-1)^{p}}{2} \frac{\pi^{-p}}{2^{n-2 p+1}} \frac{1}{n !}(-1)^{n} B_{n-p+1}, \\
f_{n, p, q} & =\frac{1-(-1)^{p+q}}{2} \frac{\pi^{-p-q}}{2^{n-2(p+q)+3}} \frac{1}{n !}(-1)^{n-q} B_{n-p-q+2},
\end{aligned}
$$


where the operators $\mathcal{L}_{0}, \mathcal{B}_{0}$ and $\tilde{c}_{p}$ are defined in the sliver frame and are related to the worldsheet energy-momentum tensor $T$, the $b$ and $c$ ghosts fields respectively. A second way of writing the solution is given in terms of wedge states with ghost insertions,

$$
\begin{aligned}
\Psi & =\lim _{N \rightarrow \infty}\left[\psi_{N}-\sum_{n=0}^{N} \partial_{n} \psi_{n}\right], \\
\psi_{n} & =\frac{2}{\pi^{2}} U_{n+2}^{\dagger} U_{n+2}\left[\left(\mathcal{B}_{0}+\mathcal{B}_{0}^{\dagger}\right) \tilde{c}\left(-\frac{\pi}{4} n\right) \tilde{c}\left(\frac{\pi}{4} n\right)+\frac{\pi}{2}\left(\tilde{c}\left(-\frac{\pi}{4} n\right)+\tilde{c}\left(\frac{\pi}{4} n\right)\right)\right]|0\rangle,
\end{aligned}
$$

where $\psi_{N}$ with $N \rightarrow \infty$ is called the phantom term $[1,5,12,13]$.

The above equations (1.1)-(1.5) allow us to write the analytic solution, either in the basis of curly $\mathcal{L}_{0}$ eigenstates or in the Virasoro $L_{0}$ eigenstates and those level expansions of the solution are very useful for the numerical evaluation of the energy. The result of the energy obtained by means of the curly $\mathcal{L}_{0}$ level expansion is given in terms of a divergent series which nevertheless can be resummed numerically by means of Padé approximants to give a good approximation of the expected value of the D-brane tension that agrees with Sen's first conjecture $[1,19]$. While in the case of using the usual Virasoro $L_{0}$ level expansion, the resulting expression for the energy seems to be a convergent series which approaches to the expected value, and therefore the use of Padé approximants was not necessary in that case $[1,17]$.

Another interesting analysis that could be performed is to derive Schnabl's analytical solution by numerical means, namely, using a similar strategy that has been employed in the case of numerical solutions constructed in other gauges like the Siegel gauge [46-51], and the so-called $a$-gauge [52-54]. Using the state space of Virasoro $L_{0}$ eigenstates, we can write a rather generic string field $\Psi$, subject to the gauge condition $\mathcal{B}_{0} \Psi=0$, called the Schnabl gauge. Truncating this string field up to some given level, we can evaluate the normalized value of the potential defined by $V(\Psi)=-S(\Psi) / T_{25}$, where $S$ is the string field theory action and $T_{25}$ represents the value of the D-brane tension. Explicitly, the normalized potential is given by

$$
V(\Psi)=2 \pi^{2}\left[\frac{1}{2}\langle\Psi, Q \Psi\rangle+\frac{1}{3}\langle\Psi, \Psi * \Psi\rangle\right] .
$$

Extremising this potential (1.6) and keeping the coefficient corresponding to the tachyon state fixed, we obtain the effective tachyon potential. Actually, we will obtain many branches for this effective tachyon potential. The configuration corresponding to Schnabl's solution can be identified with the local minimum of the branch which connects the perturbative with the non-perturbative vacuum.

Using curly $\mathcal{L}_{0}$ level-truncation computations, i.e., working out in the sliver frame, the first attempt to obtain Schnabl's solution numerically has been done in reference [55]. In the sliver frame, the level of a state is defined as the eigenvalue of the operator $\mathcal{L}_{0}+1$. For instance, the truncated level one string field, following the notation of reference [55], is given by

$$
\Psi=x_{0} \tilde{c}_{1}|0\rangle-2 x_{1}\left(\mathcal{L}_{0}+\mathcal{L}_{0}^{\dagger}\right) \tilde{c}_{1}|0\rangle-2 x_{1}\left(\mathcal{B}_{0}+\mathcal{B}_{0}^{\dagger}\right) \tilde{c}_{0} \tilde{c}_{1}|0\rangle+(\text { higher level terms }),
$$


where the coefficients of the expansion $x_{0}$ and $x_{1}$ were chosen so that $\Psi$ satisfies the Schnabl gauge. Replacing equation (1.7) into equation (1.6), we obtain the level $V^{(1,3)}\left(x_{0}, x_{1}\right)$ potential and by integrating out the coefficient $x_{1}$, namely, using $\partial_{x_{1}} V^{(1,3)}=0$, we can write the coefficient $x_{1}$ in terms of $x_{0}$, to subsequently plugging it back into the potential, $V_{\text {eff }}^{(1,3)}\left(x_{0}\right) \equiv V^{(1,3)}\left(x_{0}, x_{1}\left(x_{0}\right)\right)$, so that we are left with the effective potential which only depends on the coefficient $x_{0}$.

Since the state $\tilde{c}_{1}|0\rangle$, defined in the sliver frame, after performing the change of basis, becomes the tachyon state $c_{1}|0\rangle$, the effective potential that depends on the coefficient $x_{0}$ has been identified as the effective tachyon potential. However, we have noticed that the state $\tilde{c}_{1}|0\rangle$ is not the only one that contains the tachyon state $c_{1}|0\rangle$, for instance, the truncated level four string field contains the states $\tilde{c}_{-1}|0\rangle$ and $\tilde{c}_{-3}|0\rangle$, which after performing the change of basis, it can be shown that [1]

$$
\tilde{c}_{-1}|0\rangle=\left(c_{-1}-c_{1}\right)|0\rangle, \quad \tilde{c}_{-3}|0\rangle=\left(c_{-3}-\frac{1}{3} c_{-1}+\frac{1}{3} c_{1}\right)|0\rangle .
$$

Clearly these states also contain the tachyon state $c_{1}|0\rangle$. This observation implies that the tachyon state can get more contributions coming from states that appear at higher levels.

Leaving aside the above subtlety, and considering a truncated level five string field, like the one given in equation (1.7), it has been shown that there exist a branch of the potential which connects the perturbative with the non-perturbative vacuum and its local minimum occurs at a point where $x_{0}=0.63680186$ [55]. Note that, using equation (1.2), the analytical value of this coefficient turns out to be $x_{0, \text { exact }}=f_{0,1}=2 / \pi=0.63661977$. By computing the value of the remaining coefficients and evaluating the energy, the results of [55] suggest that the numerical solution, found by means of curly $\mathcal{L}_{0}$ level-truncation computations, seems to converge to the Schnabl's analytical solution [1].

Since the first term alone appearing in the curly $\mathcal{L}_{0}$ level expansion (1.7) does not represent the tachyon state, the effective potential depending on the single coefficient $x_{0}$ can not be identified as being the effective tachyon potential. In order to properly determine the effective tachyon potential, we must use an expansion of the string field such that the lowest state should correspond to the tachyon state alone. This calculation can be done if we express directly the string field in the Virasoro basis of $L_{0}$ eigenstates. ${ }^{1}$ For instance, up to level two states, following the notation of Sen and Zwiebach, we have

$$
\Psi=t c_{1}|0\rangle+u c_{-1}|0\rangle+v L_{-2}^{m} c_{1}|0\rangle+w b_{-2} c_{0} c_{1}|0\rangle+(\text { higher level terms })=t \mathcal{T}+\chi,
$$

where $t, u, v$ and $w$ are some unknown coefficients, and $L_{p}^{m}$ denotes the modes of the matter Virasoro operator.

We have defined the field $\mathcal{T} \equiv c_{1}|0\rangle$ as being the tachyon contribution of the string field, while $\chi$ represents the remaining terms which are linearly independent of the first term $\mathcal{T}$. To obtain the effective tachyon potential, we must integrate out the string field $\chi$, this is done by inserting the string field $\Psi$ into the potential (1.6), solving the equation of motion for $\chi$ and plugging back to the action. The resulting expression, as a function of the

\footnotetext{
${ }^{1}$ As usual, we define the level $L$ of a state as the eigenvalue of the operator $L_{0}+1$.
} 
single variable $t$ is the effective tachyon potential. Note that the effective tachyon potential computed in this way is non-unique since it depends on the choice of a specific gauge to fix the string field $\Psi$. Historically, the gauge used has been the Siegel gauge $b_{0} \Psi=0$, and the first numerical tests of Sen's first conjecture were done in this gauge [46-50]. The most recent Virasoro $L_{0}$ level-truncation computations in Siegel gauge has been performed in reference [18], where the author obtained a numerical solution up to the level $(26,78)$.

Using Virasoro $L_{0}$ level-truncation computations, we are going to derive a numerical solution $\Psi$ for tachyon condensation in Schnabl gauge $\mathcal{B}_{0} \Psi=0$. Since the operator $\mathcal{B}_{0}$ contains all even positive modes of the $b$ ghost field

$$
\mathcal{B}_{0}=b_{0}+\sum_{k=1}^{\infty} \frac{2(-1)^{k+1}}{4 k^{2}-1} b_{2 k}
$$

Schnabl gauge fixing condition turns out to be level dependent. For instance, regarding the $w$ coefficient, if we impose Schnabl gauge to the truncated level two string field (1.9), we obtain $w=0$, while using a truncated level four string field, Schnabl gauge implies that $w=-2 E / 3$, where $E$ is the coefficient in front of the state $b_{-2} c_{-2} c_{1}|0\rangle$ which appears at level four. This result is in contrast to the case of Siegel gauge, where the gauge condition $b_{0} \Psi=0$ implies that the coefficients satisfy some relations that are independent of the level of the truncated string field.

In reference [1], the author conjectured that the level dependent Schnabl gauge fixing condition would not pose problems and that the numerical high level computations of Moeller and Taylor [49] and Gaiotto and Rastelli [50] would converge to his analytical solution. One of the main motivations for writing this paper has been to test this conjecture by means of explicit numerical computations.

This paper is organized as follows. In section 2, by writing a string field $\Psi$ in terms of the elements contained in the state space of Virasoro $L_{0}$ eigenstates, we study and discuss Schnabl gauge condition $\mathcal{B}_{0} \Psi=0$, then using this string field expanded up to some level $L \leq 10$, we define and evaluate the truncated $(L, 3 L)$ potential. In section 3 , employing this truncated $(L, 3 L)$ potential, and integrating out the non-tachyonic fields, we construct the effective tachyon potential and analyze its branch structure. In section 4 , we analyze and extrapolate the data of the vacuum energy. In section 5, we study and extrapolate the data of the tachyon vev. Finally, a summary and further directions of exploration are given in the last section.

\section{Level truncation and Schnabl gauge}

To perform level-truncation computations, first we define the level $L$ of a state as the eigenvalue of the operator $L_{0}+1$. For instance, the zero momentum tachyon state $c_{1}|0\rangle$ is at level $L=0$. Let us remember that the string field action has a twist symmetry under which all coefficients of odd-twist states change sign, whereas coefficients of even-twist states remain unchanged $[46,56]$. Therefore coefficients of odd-twist states at levels above $c_{1}|0\rangle$ must always appear in the action in pairs, and they trivially satisfy the equations 
of motion if set to zero. Thus, we look for $\Psi$ containing only even-twist states. As an example, up to level six states, the truncated string field is given by

$$
\begin{aligned}
\Psi= & t c_{1}|0\rangle+u c_{-1}|0\rangle+v L_{-2}^{m} c_{1}|0\rangle+w b_{-2} c_{0} c_{1}|0\rangle+A L_{-4}^{m} c_{1}|0\rangle+B L_{-2}^{m} L_{-2}^{m} c_{1}|0\rangle \\
& +C c_{-3}|0\rangle+D b_{-3} c_{-1} c_{1}|0\rangle+E b_{-2} c_{-2} c_{1}|0\rangle+F L_{-2}^{m} c_{-1}|0\rangle+w_{1} L_{-3}^{m} c_{0}|0\rangle \\
& +w_{2} b_{-2} c_{-1} c_{0}|0\rangle+w_{3} b_{-4} c_{0} c_{1}|0\rangle+w_{4} L_{-2}^{m} b_{-2} c_{0} c_{1}|0\rangle+w_{5} c_{-5}|0\rangle+w_{6} L_{-6}^{m} c_{1}|0\rangle \\
& +w_{7} L_{-4}^{m} c_{-1}|0\rangle+w_{8} L_{-2}^{m} c_{-3}|0\rangle+w_{9} b_{-6} c_{0} c_{1}|0\rangle+w_{10} b_{-4} c_{-2} c_{1}|0\rangle+w_{11} b_{-4} c_{-1} c_{0}|0\rangle \\
& +w_{12} b_{-2} c_{-4} c_{1}|0\rangle+w_{13} b_{-2} c_{-3} c_{0}|0\rangle+w_{14} b_{-2} c_{-2} c_{-1}|0\rangle+w_{15} L_{-4}^{m} L_{-2}^{m} c_{1}|0\rangle \\
& +w_{16} L_{-2}^{m} L_{-2}^{m} c_{-1}|0\rangle+w_{17} L_{-4}^{m} b_{-2} c_{0} c_{1}|0\rangle+w_{18} L_{-2}^{m} b_{-4} c_{0} c_{1}|0\rangle+w_{19} L_{-2}^{m} b_{-2} c_{-2} c_{1}|0\rangle \\
& +w_{20} L_{-2}^{m} b_{-2} c_{-1} c_{0}|0\rangle+w_{21} L_{-2}^{m} L_{-2}^{m} L_{-2}^{m} c_{1}|0\rangle+w_{22} L_{-2}^{m} L_{-2}^{m} b_{-2} c_{0} c_{1}|0\rangle \\
& +w_{23} L_{-3}^{m} c_{-2}|0\rangle+w_{24} L_{-5}^{m} c_{0}|0\rangle+w_{25} L_{-3}^{m} L_{-2}^{m} c_{0}|0\rangle+w_{26} L_{-3}^{m} L_{-3}^{m} c_{1}|0\rangle \\
& +w_{27} b_{-3} c_{-3} c_{1}|0\rangle+w_{28} b_{-3} c_{-2} c_{0}|0\rangle+w_{29} b_{-5} c_{-1} c_{1}|0\rangle+w_{30} L_{-3}^{m} b_{-3} c_{0} c_{1}|0\rangle \\
& +w_{31} L_{-2}^{m} b_{-3} c_{-1} c_{1}|0\rangle+w_{32} b_{-3} b_{-2} c_{-1} c_{0} c_{1}|0\rangle+w_{33} L_{-3}^{m} b_{-2} c_{-1} c_{1}|0\rangle .
\end{aligned}
$$

The next step is to impose some gauge on this truncated string field. Traditionally in $L_{0}$ level-truncation computations the Siegel gauge $b_{0} \Psi=0$ has been used. Here we are going to impose another gauge, namely, the Schnabl gauge $\mathcal{B}_{0} \Psi=0$, where

$$
\mathcal{B}_{0}=b_{0}+\sum_{k=1}^{\infty} \frac{2(-1)^{k+1}}{4 k^{2}-1} b_{2 k} .
$$

As we are going to show, after imposing Schnabl gauge condition on the string field, the coefficients $t, u, v, w, \cdots$ that appear in the $L_{0}$ level expansion of the string field will satisfy some relations.

As an explicit example, let us impose the Schnabl gauge condition $\mathcal{B}_{0} \Psi=0$ on the truncated level 2 string field

$$
\Psi=t c_{1}|0\rangle+u c_{-1}|0\rangle+v L_{-2}^{m} c_{1}|0\rangle+w b_{-2} c_{0} c_{1}|0\rangle .
$$

Since the state $|0\rangle$ has the property that $b_{n}|0\rangle=0$ for $n>-2$, using the commutator and anti-commutators

$$
\left[b_{p}, L_{q}^{m}\right]=0, \quad\left\{b_{p}, b_{q}\right\}=0, \quad\left\{b_{p}, c_{q}\right\}=\delta_{p+q, 0},
$$

the computation of $\mathcal{B}_{0} \Psi$, leads to

$$
\mathcal{B}_{0} \Psi=-w b_{-2} c_{1}|0\rangle,
$$

therefore the gauge condition $\mathcal{B}_{0} \Psi=0$ implies that

$$
w=0 .
$$

Performing similar computations, if we impose the gauge condition $\mathcal{B}_{0} \Psi=0$ on a truncated level 4 string field, we get

$$
\begin{aligned}
w_{i} & =0, \quad i=1,2,3,4 . \\
w & =-\frac{2}{3} E .
\end{aligned}
$$


Note that at level 2 the relation that the coefficient $w$ satisfies is given by $w=0$, while at level 4 it has a different relation $w=-\frac{2}{3} E$. Going further, for a truncated level 6 string field, using $\mathcal{B}_{0} \Psi=0$, we can show that

$$
w=-\frac{2 E}{3}+\frac{2 w_{12}}{15}
$$

So in general, it turns out that the relation satisfied by the coefficients, after imposing the gauge condition $\mathcal{B}_{0} \Psi=0$, depends on the level of the truncated string field. This result is in contrast to the case of Siegel gauge, where the gauge condition $b_{0} \Psi=0$ implies that the coefficients satisfy some relations that are independent of the level of the truncated string field. For instance, regarding to the coefficient $w$, if we impose the Siegel gauge condition $b_{0} \Psi=0$ on a truncated level 2 string field, we obtain $w=0$. Now, if we use a truncated level 4 string field we also get $w=0$, and even for higher levels the same relation $w=0$ holds.

In reference [1], the author has conjectured that this level dependent gauge fixing would not pose problems and that the numerical high level computations of Moeller, Taylor [49], Gaiotto and Rastelli [50] would converge to his analytical solution. Extrapolating our results to higher levels, we are going to argue that the convergence of the numerical solution to the Schnabl's analytical solution [1], as $L \rightarrow \infty$, will be very slow. In this respect convergence properties of Siegel gauge is better than Schnabl gauge.

Next, let us compute the normalized value of the tachyon potential which is given by

$$
V(\Psi)=2 \pi^{2}\left[\frac{1}{2}\langle\Psi, Q \Psi\rangle+\frac{1}{3}\langle\Psi, \Psi * \Psi\rangle\right] .
$$

The $V^{(L, n)}$ level truncated potential is obtained by replacing the truncated level $L$ string field into the potential (2.10) and keeping interaction terms up to the total level $n$, note that $2 L \leqslant n \leqslant 3 L$. In this paper, we consider the maximum value of $n$, namely, we are going to work with the truncated $(L, 3 L)$ potential.

Although the computation of the cubic interaction term becomes tedious at higher levels, the evaluation of the truncated $(L, 3 L)$ potential is straightforward. Based on conservation laws [48], we have written a computer code which evaluates higher level cubic vertices. With the help of this code, we have obtained results up to level $(10,30) .^{2}$ Once we have the potential, the next step is to impose the gauge condition and then find the stationary point of the potential, where in the case of Schnabl gauge when $L \rightarrow \infty$ the stationary point should correspond to the Schnabl's analytic solution for tachyon condensation [1].

Another interesting computation that can be done with the potential is the construction of the effective tachyon potential. In order to explain the procedure for finding the effective tachyon potential, let us first set all components of the string field to zero except for the first coefficient $t$. This state will be said to be of level zero. Thus, we take

$$
\Psi=t c_{1}|0\rangle .
$$

\footnotetext{
${ }^{2}$ We would like to mention that in order to test our code, before computing the numerical solution in Schnabl gauge, we have derived the numerical solution in Siegel gauge, and shown that all our results coincide with the ones found in references $[49,50]$.
} 
Substituting (2.11) into the definition (2.10), we get the level $(0,0)$ approximation to the tachyon potential,

$$
V^{(0,0)}=2 \pi^{2}\left[-\frac{t^{2}}{2}+\frac{27 \sqrt{3} t^{3}}{64}\right] .
$$

The local minimum of the above potential is located at $t_{0}=0.456177$, and the level $(0,0)$ potential evaluated at this point has the value of $V^{(0,0)}\left(t_{0}\right)=-0.684616$.

Going to the next level, namely using the level 2 string field (2.3), and plugging it into the definition $(2.10)$, we obtain the following level $(2,6)$ potential

$$
\begin{aligned}
V^{(2,6)}= & 2 \pi^{2}\left[\frac{1}{2}\left(-t^{2}-u^{2}-6 u w+13 v^{2}+26 v w+4 w^{2}\right)\right. \\
& +\frac{1}{3}\left(\frac{81 \sqrt{3} t^{3}}{64}+\frac{99}{64} \sqrt{3} t^{2} u-\frac{585}{64} \sqrt{3} t^{2} v+\frac{19}{64} \sqrt{3} t u^{2}+\frac{\sqrt{3} u^{3}}{64}-\frac{715 t u v}{32 \sqrt{3}}\right. \\
& -\frac{9}{4} \sqrt{3} t^{2} w+\frac{3}{2} \sqrt{3} t u w+\frac{7553 t v^{2}}{64 \sqrt{3}}-\frac{1235 u^{2} v}{576 \sqrt{3}}+\frac{83083 u v^{2}}{1728 \sqrt{3}}-\frac{272363 v^{3}}{1728 \sqrt{3}} \\
& \left.\left.+\frac{65 t v w}{2 \sqrt{3}}+\sqrt{3} t w^{2}+\frac{703 u^{2} w}{108 \sqrt{3}}-\frac{65 u v w}{6 \sqrt{3}}-\frac{47 u w^{2}}{27 \sqrt{3}}-\frac{7553 v^{2} w}{108 \sqrt{3}}-\frac{65 v w^{2}}{9 \sqrt{3}}\right)\right] .
\end{aligned}
$$

Now we need to impose the gauge condition on the string field. Notice that at level 2, both the Siegel gauge condition $b_{0} \Psi=0$ and the Schnabl gauge condition $\mathcal{B}_{0} \Psi=0$ imply that $w=0$. Setting $w=0$ in the potential (2.13), we obtain the level $(2,6)$ gauge fixed potential. Since the effective tachyon potential depends on the single variable $t$ which corresponds to the tachyon coefficient, we are going to integrate out the rest of the coefficients $u$ and $v$. Using the partial derivatives of the potential (with $w=0$ ), $\partial_{u} V^{(2,6)}=0$, and $\partial_{v} V^{(2,6)}=0$, we can write the coefficients $u$ and $v$ in terms of $t$.

Starting at level $(2,6)$, coefficients other than the tachyon coefficient $t$ do not appear quadratically, therefore we cannot exactly integrate out these coefficients $u$ and $v$. We use Newton's numerical method to find the zeros of the partial derivatives of the potential. For a fixed value of the tachyon coefficient $t$, there are in general many solutions of the equations for the remaining coefficients, which correspond to different branches. The branch structure corresponding to the effective tachyon potential will be analyzed in the next section.

At this point, we are interested in the branch of the effective tachyon potential connecting the perturbative with the non-perturbative vacuum and having a minimum value which agrees with the one predicted from Sen's first conjecture. For instance, the local minimum of the level $(2,6)$ effective tachyon potential corresponds to $t_{0}=0.544204, u_{0}=$ $0.190190, v_{0}=0.055963$ and the potential evaluated at these points has the value of $V^{(2,6)}\left(t_{0}, u_{0}, v_{0}, w=0\right)=-0.959376$ which is about $96 \%$ of the exact answer.

The results for the tachyon vev and vacuum energy, up to level $(10,30)$, are shown in tables 1 and 2. As we can see, our results in Siegel gauge are the same as the ones given in references $[49,50]$. Note that, in Schnabl gauge, at level $L=6$ the energy overshoots the predicted analytical answer of -1 and appears to further decrease at higher levels. In the case of Siegel gauge, this phenomenon happens at level $L=14$ [50]. As a first impression, 


\begin{tabular}{|c|c|c|}
\hline$L$ & $c_{1}|0\rangle$ & $E^{\mathrm{Sie}}$ \\
\hline 0 & 0.456177990470 & -0.684616159915 \\
\hline 2 & 0.544204232320 & -0.959376599521 \\
\hline 4 & 0.548398986499 & -0.987821756244 \\
\hline 6 & 0.547932362586 & -0.995177120537 \\
\hline 8 & 0.547052407685 & -0.997930183378 \\
\hline 10 & 0.546260900230 & -0.999182458475 \\
\hline
\end{tabular}

Table 1. $(L, 3 L)$ level-truncation results for the tachyon vev and vacuum energy in Siegel gauge.

\begin{tabular}{|c|c|c|}
\hline$L$ & $c_{1}|0\rangle$ & $E^{\text {Sch }}$ \\
\hline 0 & 0.456177990470 & -0.684616159915 \\
\hline 2 & 0.544204232320 & -0.959376599521 \\
\hline 4 & 0.548938521247 & -0.994651904750 \\
\hline 6 & 0.548315148955 & -1.003983765388 \\
\hline 8 & 0.547321883647 & -1.007110280219 \\
\hline 10 & 0.546508411314 & -1.008189759705 \\
\hline
\end{tabular}

Table 2. $(L, 3 L)$ level-truncation results for the tachyon vev and vacuum energy in Schnabl gauge.

it seems that the level-truncation procedure is breaking down, in the case of Schnabl gauge for $L \geq 6$, and in the case of Siegel gauge for $L \geq 14$. Nevertheless, by using a clever extrapolation technique to level-truncation data obtained in Siegel gauge for $L<18$ to estimate the vacuum energies even for $L>18$, in reference [50], the authors have shown that the results may simply indicate that the approach of the energy to -1 as $L \rightarrow \infty$ is non-monotonic, actually it is predicted that the energy reaches a minimum value for $L \sim 27$, but then turns back to approach asymptotically -1 for $L \rightarrow \infty$.

In the case of Schnabl gauge, applying Gaiotto-Rastelli extrapolation and an alternative technique called as Padé extrapolation to the level-truncation data for $L \leq 10$ to estimate the vacuum energies even for $L>10$, in section 4 , we are going to predict that the energy reaches a minimum value for $L \sim 12$, and then turns back to approach asymptotically -1 for $L \rightarrow \infty$.

For the case of the tachyon vev data obtained in Schnabl gauge which is given in table 2. Note that the value of the tachyon vev gets a maximum value near level $L=4$ and then starts to decrease. In order to reach the analytical value of 0.553465 [1], there should be some higher value of $L>4$ such that the tachyon vev stops decreasing and then starts increasing to approach asymptotically the expected result. In the case of Siegel gauge, it may also happen that there is some value of $L>4$ such that the tachyon vev stops decreasing. Actually, this kind of possibility has been considered in reference [61], where the analytic value $\sqrt{3} / \pi \cong 0.551328$ was conjectured for the tachyon vev. All these issues related to the discussion of the tachyon vev will be analyzed in section 5 . 


\section{Effective tachyon potential and its branch structure}

Using the $(L, 3 L)$ level truncated potential with $L=2,4,6$ and 8 , in this section, we are going to analyze the branch structure of the effective tachyon potential derived in Siegel as well as in Schnabl gauge.

\subsection{Branch structure in Siegel gauge}

As we have seen in the previous section, the truncated level $L$ string field can be written as $\Psi=t \mathcal{T}+\chi$, where $\mathcal{T} \equiv c_{1}|0\rangle$ represents the tachyonic part and $\chi$ is the remaining non-tachyonic contribution. By substituting this $L_{0}$ level expansion of $\Psi$ into the string field action, we derive the $V^{(L, 3 L)}$ level truncated potential. Note that this potential will depend on the tachyonic coefficient $t$ as well as on the other non-tachyonic coefficients that are contained in the $\chi$ term. In this subsection, using the Siegel gauge condition $b_{0} \Psi=0$, we are going to study the effective tachyon potential.

To construct the effective tachyon potential which depends only on the tachyon coefficient $t$, we must integrate out the non-tachyonic coefficients. Since starting at level $(2,6)$, coefficients other than the tachyon coefficient $t$ do not appear quadratically, we cannot exactly integrate out these non-tachyonic coefficients. Therefore, we are forced to use numerical methods to study the effective tachyon potential. We have used Newton's method to find the zeros of the partial derivatives of the potential. For a fixed value of the tachyon coefficient $t$, there are many solutions of the equations for the remaining coefficients, which correspond to the different branches of the effective tachyon potential.

We are interested in the branches that are close to the physical branch, namely, the branch connecting the perturbative with the non-perturbative vacuum which we label as branch 1 for Siegel gauge and branch $A$ for Schnabl gauge. In the case of Siegel gauge, we have found four roots of the level $(2,6)$ potential that correspond to four branches label by 1,2,3 and 4, where branch 1 precisely corresponds to the physical branch. To analyze these branches 1, 2 and 3 at higher levels $L>2$, we have used those roots found at level $L=2$ as initial values, while to derive branch 4 at higher levels, it has been necessary to find the corresponding root of the level $(4,12)$ potential.

Figure 1 shows these four branches of the effective potential at levels $(2,6),(4,12),(6,18)$ and $(8,24)$. The physical branch (branch 1 ) has the interesting property that it meets branches 2 and 4 at points where Newton's method becomes unstable. The location of these points denoted as $t_{-}$and $t_{+}$depends on the level and are given in table 3 . At level $(2,6)$ it happens near $t_{-} \approx-0.17$ where branch 1 meets branch 2 from the left, and $t_{+} \approx 3.34$ where branch 1 meets branch 4 from the right. As we increase the level of the potential, we noticed that these two points converge to some fixed values, furthermore it seems that branches 2,3 and 4 are getting closer to branch 1 in a smooth way. These properties related to the branch structure where already discussed by Moeller and Taylor [49], even though they have not identified branch 4 which meets branch 1 for positive values of the tachyon coefficient, they have mentioned the possible existence of such a branch. 


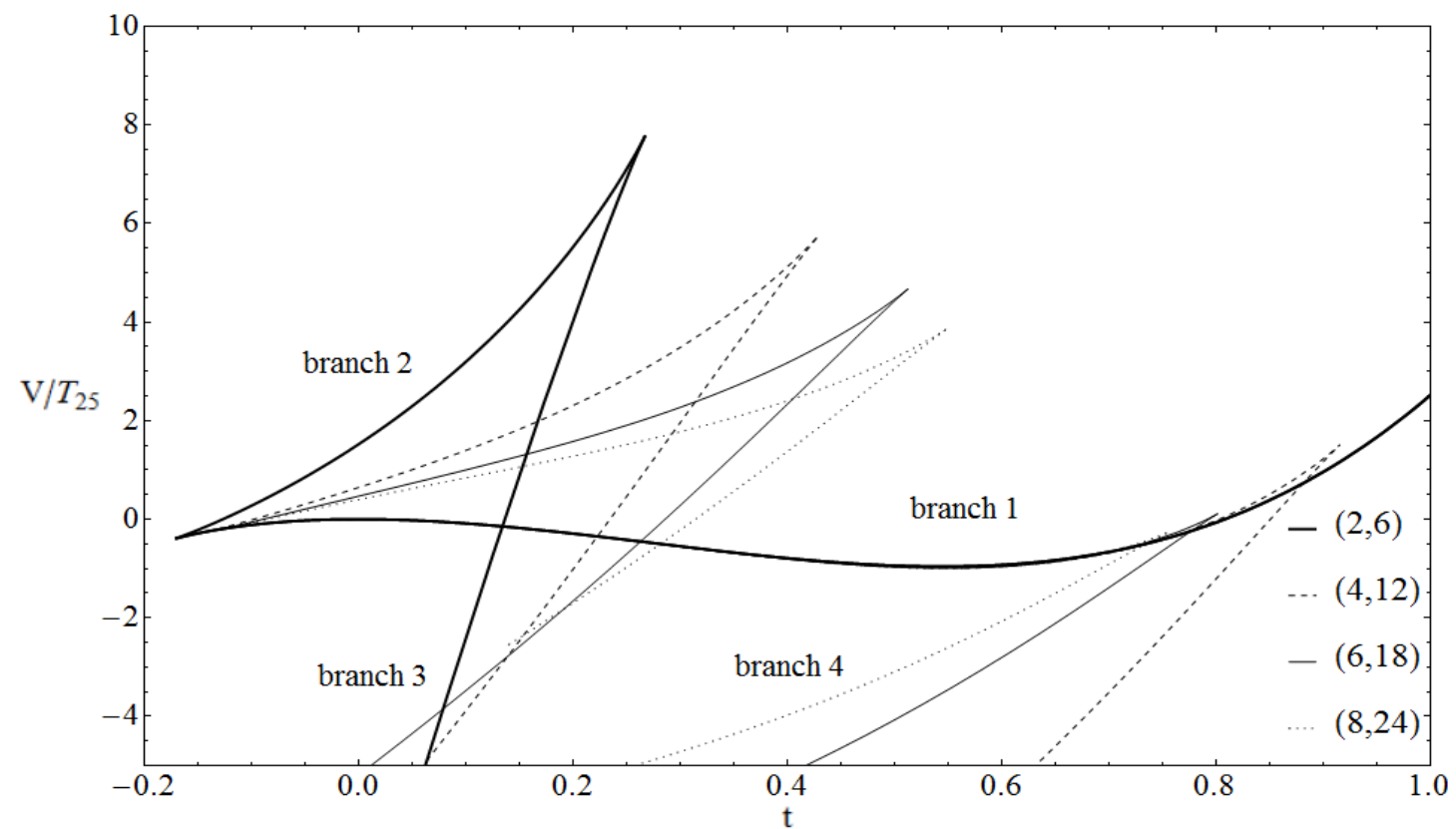

Figure 1. Plot of branches 1, 2, 3 and 4 corresponding to the effective tachyon potential at levels $(L, 3 L)$ with $L=2,4,6$ and 8 , in Siegel gauge.

\begin{tabular}{|l|r|r|r|r|}
\hline & \multicolumn{1}{|c|}{$L=2$} & \multicolumn{1}{c|}{$L=4$} & \multicolumn{1}{c|}{$L=6$} & \multicolumn{1}{c|}{$L=8$} \\
\hline$t_{-}$ & -0.1734 & -0.1428 & -0.1336 & -0.1292 \\
$t_{+}$ & 3.3468 & 0.9149 & 0.8012 & 0.7549 \\
\hline
\end{tabular}

Table 3. Approximate values of the tachyon coefficients $t$ where Newton's algorithm stops converging for branch 1 at levels $(L, 3 L)$ in Siegel gauge.

\subsection{Branch structure in Schnabl gauge}

The $(L, 3 L)$ potential in Schnabl gauge can be constructed after imposing the so-called Schnabl gauge condition $\mathcal{B}_{0} \Psi=0$ on the string field $\Psi$. The $(L, 3 L)$ effective tachyon potential is then obtained after integrating out the non-tachyonic coefficients that appear in the expansion of $\Psi$. We have seen that the gauge condition implies some relations that these non-tachyonic coefficients must obey. It turns out that up to level $L=2$, either Siegel or Schnabl gauge condition implies the same relations for the non-tachyonic coefficients, such that up to this level, the branch structure of the effective tachyon potential in Schnabl gauge is exactly the same as in Siegel gauge.

Starting at level $L=4$, Schnabl gauge condition provides relations for the nontachyonic coefficients that are different from the ones obtained in Siegel gauge. Therefore, in the case of Schnabl gauge, we expect for levels $L \geq 4$ a different branch structure for the effective tachyon potential as compared to Siegel gauge. And in fact, we cannot extend the branches found at level $L=2$ to higher levels (with the exception of the physical branch). For instance, if we try to extend these branches to level $L=4$, using as initial values for Newton's method the zeros found from the partial derivatives of the level $(2,6)$ potential, 


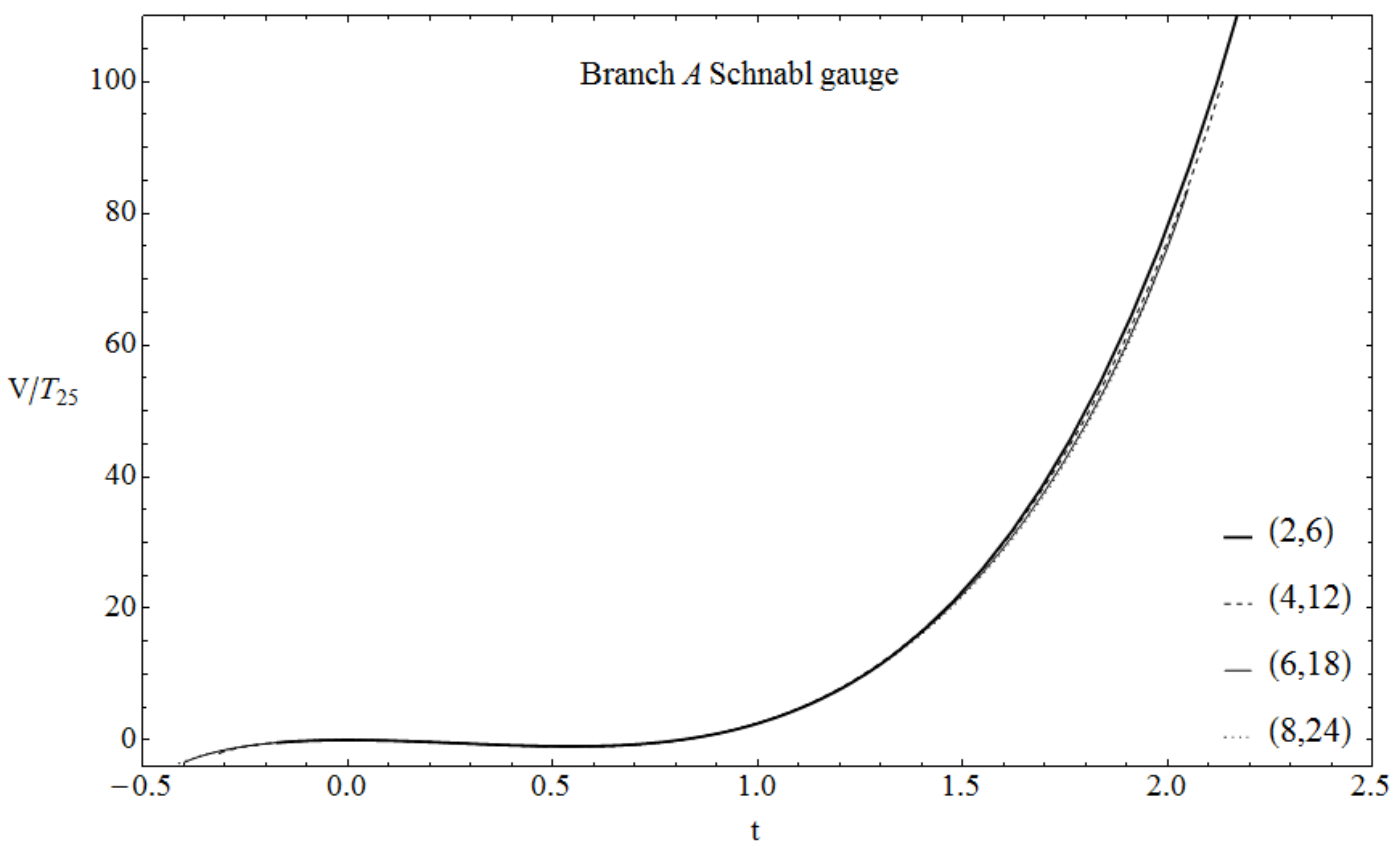

Figure 2. Branch $A$ of the effective tachyon potential in Schnabl gauge at different truncation levels.

we discover that the algorithm converges to a single solution which precisely corresponds to the physical branch.

Thus, to study properly the branch structure of the effective tachyon potential in Schnabl gauge, for a fixed value of the tachyon coefficient $t$, we have been required to obtain all the zeros of the partial derivatives of the level $(4,12)$ potential. As a result, we have found many different solutions (branches) including the physical branch (named as branch $A$ ), most of these solutions have energy scales that are far away from the physical branch. For a matter of analysis, we will be interested in branches that are close to the physical branch.

In figure 2, we show the plot of branch $A$ at different truncation levels. Note that, each time we increase the level, the profile of branch $A$ does not change significantly and its shape looks quite similar to branch 1 of Siegel gauge. We have also observed that the numerical algorithm used to construct Branch $A$ fails to converge outside some region defined by $t_{-}<t<t_{+}$. As shown in table 4 , the locations of the points $t_{-}$and $t_{+}$depend on the level and appear to converge under level-truncation to fixed values. For a better understanding of what happens near these points and verify if they have the same origin as in the case of Siegel gauge, we are going to study the structure of other branches that are close to branch $A$.

We have discovered three different branches that are near branch $A$, one of these branches (named as branch $D$ ) does not intercept any of the other two branches at least in the region where $t \in\left(t_{-}, t_{+}\right)$and extends beyond this region of interest. In relation to the other two branches (named as branch $B$ and branch $C$ ), we observe that branch $B$ 


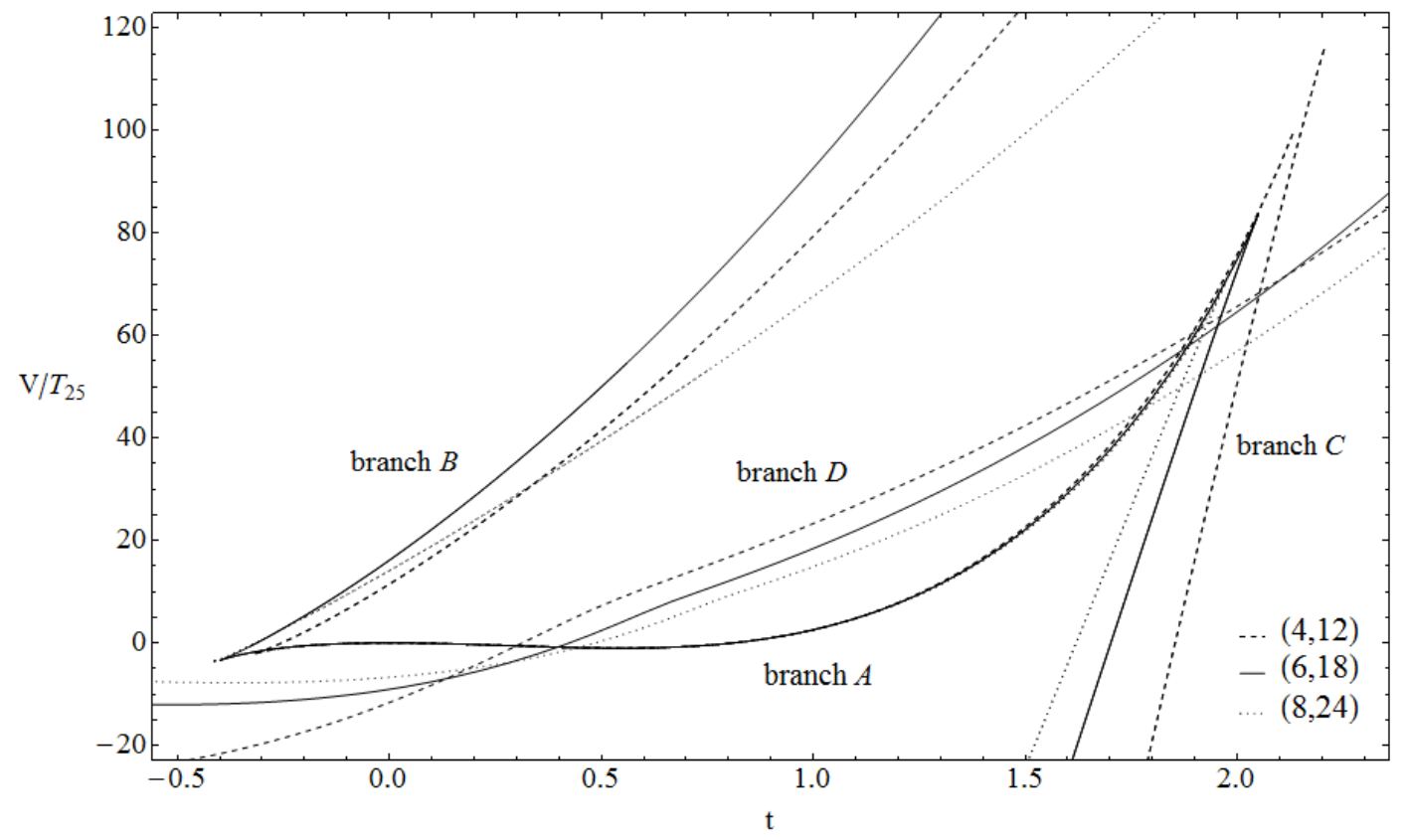

Figure 3. Plot of branches $A, B, C$ and $D$ corresponding to the effective tachyon potential at levels $(L, 3 L)$ with $L=4,6$ and 8, in Schnabl gauge.

\begin{tabular}{|l|r|r|r|r|}
\hline & \multicolumn{1}{|c|}{$L=4$} & \multicolumn{1}{c|}{$L=6$} & \multicolumn{1}{c|}{$L=8$} & \multicolumn{1}{c|}{$L=10$} \\
\hline$t_{-}$ & -0.3139 & -0.3992 & -0.4124 & -0.4121 \\
$t_{+}$ & 2.2046 & 2.0496 & 1.9712 & 1.9257 \\
\hline
\end{tabular}

Table 4. Approximate values of the tachyon coefficients $t$ where Newton's algorithm stops converging for branch $A$ at levels $(L, 3 L)$ in Schnabl gauge.

intercept branch $A$ at the point $t_{-}$, and branch $C$ intercepts branch $A$ at the point $t_{+}$. These branches $B, C$ and $D$ together with the branch $A$ are shown in figure 3 .

Regarding the construction of branches $B$ and $C$, it turns out that for given initial values of the non-tachyonic coefficients, Newton's method has a limited region of convergence, therefore to probe these branches in the region of interest defined by $\left(t_{-}, t_{+}\right)$, we were required to use diverse initial values for the non-tachyonic coefficients corresponding to different fixed values of $t \in\left(t_{-}, t_{+}\right)$. We would like to point out that branches $B$ and $C$ play similar roles as branches 2 and 4 of Siegel gauge case, namely, these branches $B$ and $C$ intercept branch $A$ at the points where the numerical algorithm used to construct branch $A$ becomes unstable.

Another observation concerns how the structure of branches $B$ and $C$ changes with the level. Branches $B$ and $C$ appear to approach under level-truncation to branch $A$. When we move from level $(4,12)$ to $(6,18)$, the slope of branch $B$ increases its value, whereas when the level change from $(6,18)$ to $(8,24)$, the slope of branch $B$ significantly decreases its value, so that branch $B$ seems to move towards branch $A$. Regarding branch $C$, as shown in figure 3 , the change of its slope seems to have a smooth behavior towards branch 
$A$, this behavior is similar to branch 4 of Siegel gauge. It would be interesting to analyze the behavior of these branches at levels $(L, 3 L)$ with $L>10$, however, at higher levels to obtain only a few points along these branches should demand a lot of computing time.

Let us comment about the region $\left(t_{-}, t_{+}\right)$where the numerical algorithm used to construct branch $A$ brings convergent results. In the case of Siegel gauge, as shown in table 3 , this region seems to converge approximately to $(-0.1,0.7)$, while in the case of Schnabl gauge, it converges to $(-0.4,1.9)$. From a mathematical point of view, it turns out that the existence of the points $t_{-}$and $t_{+}$is related to the presence of other branches which intercept the physical branch at these points.

Regarding the physical interpretation of these branch points, let us mention that in background independent string field theory, the tachyon potential can be shown to have the form $V(T)=(1+T) e^{-T}[57-59]$. While the field $T$ is related to $t$ through a nontrivial field redefinition, it is clear that the potential $V$ is unbounded below as $T \rightarrow-\infty$, and contains no branch points to the left of the stable vacuum. Thus, the branch point found for negative values of $t$ is not physical. And in fact, in the case of Siegel gauge, there is a strong evidence that the two branch points $t_{-}$and $t_{+}$appearing in the numerical computation of the effective tachyon potential are gauge artifacts arising when the field configuration along the effective potential leaves the region of validity of the gauge condition [60]. If the analysis of reference [60] can be extended to generic gauges, our results suggest that the region of validity of Schnabl gauge is bigger than the region of validity of Siegel gauge.

\section{Extrapolation techniques and the vacuum energy analysis}

Using direct $(L, 3 L)$ level-truncation computations, we have constructed the effective tachyon potential in Schnabl gauge up to level $L=10$. Selecting the branch of the potential that connects the perturbative with the non-perturbative vacuum and computing its local minimum, we have determined the vacuum energy. As we can see from table 2, starting at level $L=6$, the data for the energy overshoots the conjectured value of the normalized brane tension.

According to the data up to level $L=10$, it seems that the energy will continue to decrease. We would like to know if this pattern will be preserved at higher levels, namely, for levels $L>10$ the energy continues to decrease, or as in the case of Siegel gauge [50] it may happen that at some level $L_{\text {min }}>10$ the energy reaches a minimum value and then starts increasing to approach asymptotically, as $L \rightarrow \infty$, the expected value of -1 . These issues could be answered if, of course, we would have available data for levels $L>10$. Although we do not have this data, by extrapolating the known results we already have up to level $L=10$, we can predict the values of the energy for levels $L>10$ which should correspond (with a good degree of approximation) to the values obtained by means of messy direct $(L, 3 L)$ level-truncation computations.

A clever extrapolation method, which we refer as Gaiotto-Rastelli extrapolation technique, has been proposed in reference [50]. In the case of Siegel gauge, this technique has been successfully used to predict the values of the energy for levels $L>18$. In this section, after a brief review of Gaiotto-Rastelli technique, we are going to analyze our known data 
for the energy in Schnabl gauge obtained up to level $L=10$. Then, we will study another extrapolation method, and since the function that will be used to interpolate the known values of the energy will be a rational function in $L$, this method will be called as Padé extrapolation technique.

\subsection{Gaiotto-Rastelli extrapolation technique}

In the case of Siegel gauge, in reference [50] using direct $(L, 3 L)$ level-truncation computations, the values of the energy up to level $L=18$ were obtained, and it has been shown that at level $L=14$ the data overshoots the expected value of -1 . As a first impression, it seems that the level-truncation procedure is breaking down for $L \geq 14$. Nevertheless, employing a clever extrapolation technique to level-truncation data for $L \leq 18$ to estimate the vacuum energies even for $L>18$, Gaiotto and Rastelli have shown that the results may simply indicate that the approach of the energy to -1 as $L \rightarrow \infty$ is non-monotonic, and actually it is predicted that the energy reaches a minimum value for $L \sim 27$, but then turns back to approach asymptotically -1 for $L \rightarrow \infty$.

Gaiotto-Rastelli extrapolation technique used the information of the effective tachyon potentials $V_{L}(t)$. We have determined these potentials up to level $L=10$ in Siegel as well as in Schnabl gauges by means of $(L, 3 L)$ level-truncation computations. A detailed discussion related to the effective tachyon potential has been given in section 3 , where we have analyzed the branch structure of this potential. In this section, we are going to work with the physical branch, ${ }^{3}$ namely, the branch that connects the perturbative with the non-perturbative vacuum. In the case of Schnabl gauge, this physical branch is important because when $L \rightarrow \infty$ its local minimum should correspond to the analytical solution found by Schnabl [1].

Given the effective tachyon potentials derived in some gauge $V_{i}(t)$ with $i=0,2, \cdots, L$, the interpolating potential is defined as

$$
V_{L}^{M}(t)=\sum_{n=0}^{M / 2} \frac{a_{n}(t)}{(L+1)^{n}},
$$

where $M$ indicates the degree of the interpolation. As we can see, the value $1+M / 2$ is equal to the number of effective potentials contained in the set $\left\{V_{0}(t), V_{2}(t), V_{4}(t), \ldots, V_{L}(t)\right\}$ and the functions $a_{n}(t)$ can be expressed as linear combinations of these potentials $V_{i}(t)$.

Before constructing the interpolating potentials $V_{L}^{M}(t)$ in Schnabl gauge, using the effective potentials in Siegel gauge $V_{i}^{\mathrm{Sie}}(t)$, we are going to construct $V_{L}^{M}(t)$. We consider first the case of Siegel gauge, since in order to test and validate our computations, we would like to compare our results with the well known results obtained in reference [50].

\subsubsection{Siegel gauge}

As a pedagogical illustration, let us construct $V_{L}^{M}(t)$ with $M=4$ in Siegel gauge

$$
V_{L}^{4}(t)=\sum_{n=0}^{2} \frac{a_{n}(t)}{(L+1)^{n}} .
$$

\footnotetext{
${ }^{3}$ Remember that this physical branch has been labelled as branch 1, and branch $A$, for Siegel, and Schnabl gauge respectively.
} 
Note that for this value of $M$, we need $1+M / 2=3$ entries, that is, the first three effective potentials (up to level $L=4$ ): $\left\{V_{0}^{\mathrm{Sie}}(t), V_{2}^{\mathrm{Sie}}(t), V_{4}^{\mathrm{Sie}}(t)\right\}$. To obtain the coefficients $a_{0}(t)$, $a_{1}(t)$ and $a_{2}(t)$, we evaluate the potential $V_{L}^{4}(t)$ defined in (4.2) at $L=0,2,4$ and equate the result to the known effective potentials, namely

$$
\begin{aligned}
V_{0}^{4}(t) & =V_{0}^{\mathrm{Sie}}(t), \\
V_{2}^{4}(t) & =V_{2}^{\mathrm{Sie}}(t), \\
V_{4}^{4}(t) & =V_{4}^{\mathrm{Sie}}(t) .
\end{aligned}
$$

Solving the above system of equations, we can obtain the $a_{n}(t)$ coefficients as linear combinations of the potentials $V_{i}^{\mathrm{Sie}}(t)$

$$
\begin{aligned}
& a_{0}(t)=\frac{1}{8}\left[V_{0}^{\mathrm{Sie}}(t)-18 V_{2}^{\mathrm{Sie}}(t)+25 V_{4}^{\mathrm{Sie}}(t)\right], \\
& a_{1}(t)=-V_{0}^{\mathrm{Sie}}(t)+\frac{1}{2}\left[27 V_{2}^{\mathrm{Sie}}(t)-25 V_{4}^{\mathrm{Sie}}(t)\right], \\
& a_{2}(t)=\frac{1}{8}\left[15 V_{0}^{\mathrm{Sie}}(t)-90 V_{2}^{\mathrm{Sie}}(t)+75 V_{4}^{\mathrm{Sie}}(t)\right]
\end{aligned}
$$

Plugging these coefficients (4.6)-(4.8) into equation (4.2), we obtain the interpolating potential $V_{L}^{4}(t)$ in Siegel gauge

$$
V_{L}^{4}(t)=\frac{\left(L^{2}-6 L+8\right) V_{0}^{\mathrm{Sie}}(t)+L\left(25(L-2) V_{4}^{\mathrm{Sie}}(t)-18(L-4) V_{2}^{\mathrm{Sie}}(t)\right)}{8(L+1)^{2}} .
$$

We can use the above potential $V_{L}^{4}(t)$ to extrapolate the values of the energy for levels $L>4$. For instance, at level $L=6$, the minimum value of $V_{6}^{4}(t)$ happens at the point where $t_{0}=0.548497$ and the value of $V_{6}^{4}(t)$ evaluated at this point gives

$$
V_{6}^{4}\left(t_{0}\right)=-0.995462 \text {. }
$$

This result (4.10) exactly matches the result found in reference [50]. Note that the direct $(L, 3 L)$ level-truncation computation (with $L=6$ ) brings the value of -0.995177 for the vacuum energy in Siegel gauge.

By following the same procedures shown above, we can obtain the interpolating potentials $V_{L}^{M}(t)$ for $M=6,8,10$. It turns out that our results are identical to the results presented in reference [50]. Once we have learned the method of Gaiotto-Rastelli extrapolation technique and validated our results in Siegel gauge, we move to the case of interest, namely, Schnabl gauge.

\subsubsection{Schnabl gauge}

To construct the interpolating potentials $V_{L}^{M}(t)$ in Schnabl gauge, we follow the procedures explained in the case of Siegel gauge. As a matter of illustration, the interpolating potential $V_{L}^{4}(t)$ looks similar to the one obtained in Siegel gauge (4.9). Actually we only need to perform the replacement $V_{L}^{\mathrm{Sie}}(t) \rightarrow V_{L}^{\mathrm{Sch}}(t)$, so that

$$
V_{L}^{4}(t)=\frac{\left(L^{2}-6 L+8\right) V_{0}^{\mathrm{Sch}}(t)+L\left(25(L-2) V_{4}^{\mathrm{Sch}}(t)-18(L-4) V_{2}^{\mathrm{Sch}}(t)\right)}{8(L+1)^{2}} .
$$




\begin{tabular}{|c|c|c|c|c|}
\hline & $L=4$ & $L=6$ & $L=8$ & $L=10$ \\
\hline$M=4$ & -0.99465190 & -1.00592012 & -1.01115471 & -1.01410566 \\
$M=6$ & & -1.00398376 & -1.00753539 & -1.00918262 \\
$M=8$ & & & -1.00711028 & -1.00822786 \\
$M=10$ & & & & -1.00818975 \\
\hline & $L=12$ & $L=14$ & $L=16$ & $L=18$ \\
\hline$M=4$ & -1.01597655 & -1.01725962 & -1.01819029 & -1.01889426 \\
$M=6$ & -1.01004504 & -1.01053404 & -1.01082671 & -1.01100837 \\
$M=8$ & -1.00857415 & -1.00859518 & -1.00847386 & -1.00829192 \\
$M=10$ & -1.00847289 & -1.00841901 & -1.00821931 & -1.00796005 \\
\hline
\end{tabular}

Table 5. Predicted values of the energy $E^{M}(L)$ obtained from the interpolating potentials $V_{L}^{M}(t)$ in Schnabl gauge, at various orders of $M$ and for $L \leq 18$. In the top half of the table, the value of the diagonal entries $E^{M=L}(L)$ coincide with the direct $(L, 3 L)$ level-truncation computations.

By computing the local minimum of the potential (4.11) for values of $L>4$, we can determine the extrapolated values of the energy. For instance, at level $L=6$, the minimum value of $V_{6}^{4}(t)$ happens at the point where $t_{0}=0.549303$, and evaluating $V_{6}^{4}(t)$ at this point gives the prediction $V_{6}^{4}\left(t_{0}\right)=E^{4}(6)=-1.005920$ for the energy at level 6 . The direct $(L, 3 L)$ level-truncation computation (with $L=6$ ) brings the value of $E(6)=-1.003983$.

Since we know the effective potentials in Schnabl gauge $\left\{V_{0}^{\mathrm{Sch}}(t), V_{2}^{\mathrm{Sch}}(t), \cdots, V_{10}^{\mathrm{Sch}}(t)\right\}$ up to level $L=10$, we have determined the interpolating potentials $V_{L}^{M}(t)$ up the maximum value of $M=10$, and by computing the local minimum of these potentials which happens at a point close to $t_{0} \sim 0.54$, we can predict the values of the energy $V_{L}^{M}\left(t_{0}\right)=E^{M}(L)$. Some results of these computations are shown in table 5 .

As we can see from table 5 , the predicted values for the energy obtained by means of these interpolating potentials $V_{L}^{M}(t)$ are close to the values obtained by direct $(L, 3 L)$ level-truncation computations. Note also that as we increase the value of $M$, the degree of approximation improves. For instance, using $M=8$, namely by only knowing leveltruncation results up to level 8 , one can obtain the prediction $E^{8}(10)=-1.008227$ for the energy at level 10 , to be compared to the value $E(10)=-1.008189$ which has been obtained by direct $(10,30)$ level-truncation computation.

By analyzing the predicted values of the energy $E^{M}(L)$ as a function of the level, we observed that for $M>4$ the function $E^{M}(L)$ behaves non-monotonically, namely, as we increase the value of the level, the function $E^{M}(L)$ decreases until reaching a minimum value $E^{M}\left(L_{\min }\right)$, then for values of the level such that $L>L_{\min }$ the function $E^{M}(L)$ starts increasing and approaches asymptotically a value close to -1 . The plot of $E^{M}(L)$ with $M=10$, which is shown in figure 4, illustrates clearly this point.

Using $E^{M}(L)$ with values of $M=6,8,10$, we have found the corresponding values of $L_{\text {min }}$, and $E^{M}\left(L_{\text {min }}\right)$ together with the asymptotic one $E^{M}(L \rightarrow \infty)$, the results are shown in table 6 . By extrapolating the level-truncation data for $L \leq 10$ to estimate the vacuum energies for $L>10$, we predict that the energy reaches a minimum value at $L \sim 12$, and 


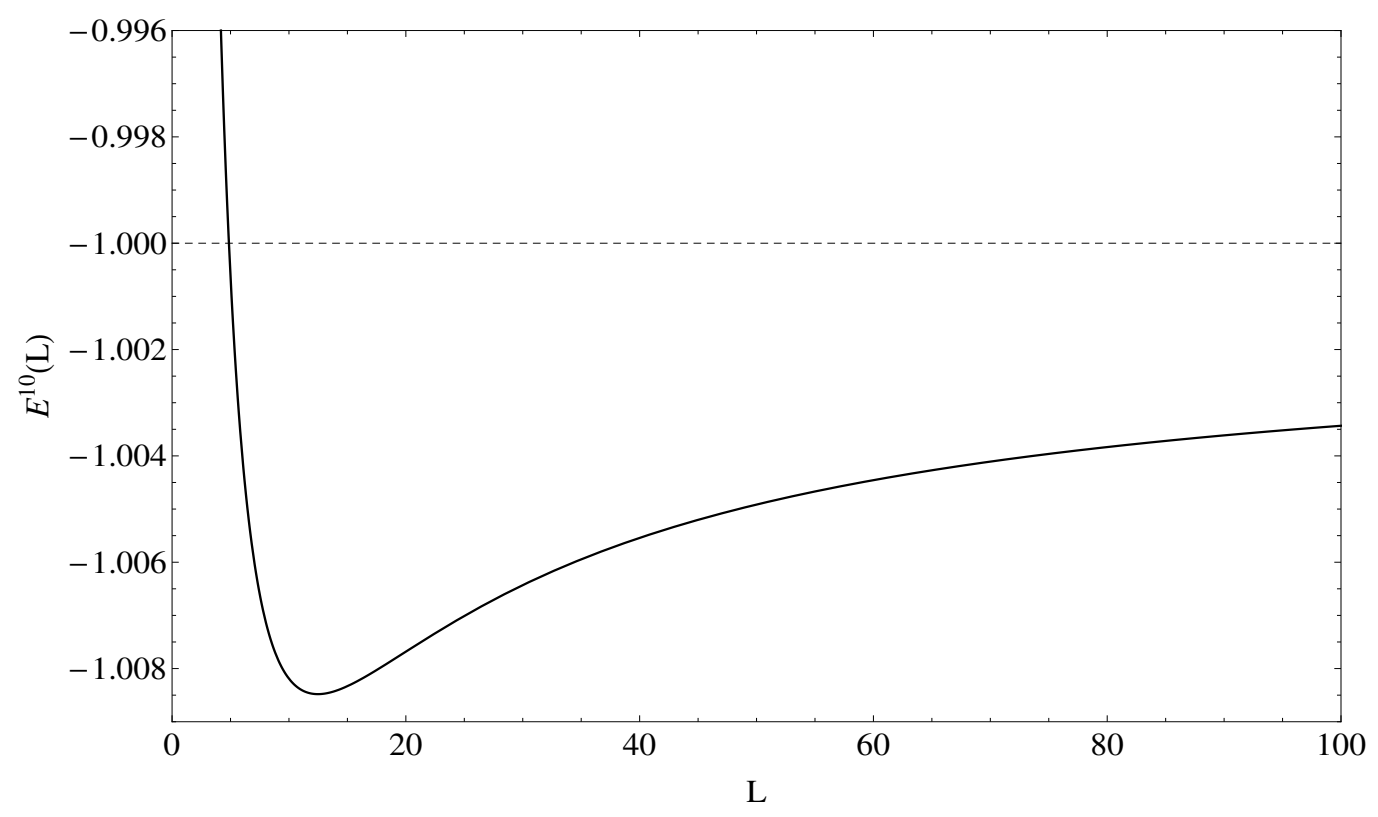

Figure 4. Plot of $E^{M}(L)$ with $M=10$, for the energy in Schnabl gauge as a function of the level. The dashed line represents the analytical value -1 .

\begin{tabular}{|c|c|c|c|}
\hline$M$ & $L_{\min }$ & $E^{M}\left(L_{\min }\right)$ & $E^{M}(L \rightarrow \infty)$ \\
\hline 6 & 32.98 & -1.00398376 & -1.01083980 \\
8 & 13.14 & -1.00711028 & -1.00348070 \\
10 & 12.47 & -1.00818977 & -1.00161871 \\
\hline
\end{tabular}

Table 6. The local minimum $E^{M}\left(L_{\text {min }}\right)$ and the asymptotic $E^{M}(L \rightarrow \infty)$ value of $E^{M}(L)$ for $M=6,8,10$ in Schnabl gauge.

then turns back to approach asymptotically -1 as $L \rightarrow \infty$. It should be nice to confirm this prediction by means of direct $(L, 3 L)$ level-truncation computations.

In order to bring an additional support for the previous predicted minimum value of the energy that should happen at $L \sim 12$, in the next subsection, we are going to use the well known results in Siegel gauge to test another method of extrapolation technique which will then be applied to the case of Schnabl gauge.

\subsection{Padé extrapolation technique}

Instead of using the set of effective tachyon potentials $V_{i}(t)$ with $i=0,2, \cdots, L$, we are going to use the values of the local minimum of these potentials, namely, the values of the vacuum energy which up to level $L=10$ are given in tables 1 and 2 for Siegel and Schnabl gauges respectively.

The derivation of the vacuum energy by means of $(L, 3 L)$ level-truncation computations is computationally less cumbersome than the construction of the corresponding effective tachyon potential. So in this sense, an extrapolation method that uses the data of the vacuum energy instead of the effective potential should be much simpler. 
Given the data of the vacuum energy up to some level $E(0), E(2), \cdots, E(L)$ derived in some gauge together with the asymptotic expected value $E(L \rightarrow \infty)=-1$, we define the following interpolating rational function

$$
f_{N}(L)=\frac{\sum_{n=0}^{N} a_{n} L^{n}}{1+\sum_{n=1}^{N} b_{n} L^{n}}
$$

where $N$ indicates the degree of the interpolation. The value $2 N+1$ is equal to the number of elements contained in the set $\{E(0), E(2), \cdots, E(L)\} \cup\{E(L \rightarrow \infty)=-1\}$. As we are going to show by means of an explicit example, the coefficients $a_{n}$ and $b_{n}$ can be determined in terms of the data points.

Using the data of the vacuum energy in Siegel gauge up to level $L=10$, we will construct these interpolating functions $f_{N}(L)$. We consider first the case of Siegel gauge, because in order to test the Padé extrapolation method, we should compare our predicted results with the well known results obtained in reference [50].

\subsubsection{Siegel gauge}

As an explicit example, let us construct $f_{N}(L)$ with $N=1$ in Siegel gauge

$$
f_{1}(L)=\frac{a_{0}+a_{1} L}{1+b_{1} L}
$$

for this value of $N$, we need $2 N+1=3$ entries, that is the first two vacuum energies $E^{\mathrm{Sie}}(0), E^{\mathrm{Sie}}(2)$ together with the asymptotic value -1 . To obtain the coefficients $a_{0}, a_{1}$ and $b_{1}$, we evaluate the function $f_{1}(L)$ defined in (4.13) at $L=0,2, \infty$ and equate the results to the known values, namely

$$
\begin{aligned}
f_{1}(0) & =E^{\mathrm{Sie}}(0)=-0.68461615991569, \\
f_{1}(2) & =E^{\mathrm{Sie}}(2)=-0.95937659952124, \\
f_{1}(L \rightarrow \infty) & =\frac{a_{1}}{b_{1}}=-1 .
\end{aligned}
$$

Solving the above system of equations, we can obtain the $a_{0}, a_{1}$ and $b_{1}$ coefficients

$$
a_{0}=-0.68461615991569, \quad a_{1}=-3.38180010003359, \quad b_{1}=3.38180010003359 .
$$

Substituting these coefficients (4.17) into equation (4.13), we obtain the interpolating function $f_{1}(L)$ of order $N=1$ in Siegel gauge.

Using this function $f_{1}(L)$, we can extrapolate the values of the energy for levels $L>2$. For instance, evaluating this function at level $L=4$, we have

$$
f_{1}(4)=-0.97829011
$$

The $(L, 3 L)$ level-truncation computation (with $L=4$ ) brings the value of -0.98782175 for the vacuum energy in Siegel gauge. It turns out that as we increase the value of $N$, the degree of approximation becomes better. 


\begin{tabular}{|c|c|c|c|c|}
\hline & $L=4$ & $L=6$ & $L=8$ & $L=10$ \\
\hline$N=1$ & -0.9782901157 & -0.9851868492 & -0.9887581331 & -0.9909419314 \\
$N=2$ & & -0.9951771205 & -0.9979360018 & -0.9991909062 \\
$N=3$ & & & & -0.9991824585 \\
\hline & $L=12$ & $L=14$ & $L=16$ & $L=18$ \\
\hline$N=1$ & -0.9924153031 & -0.9934764189 & -0.9942770695 & -0.9949026734 \\
$N=2$ & -0.9998305359 & -1.0001802977 & -1.0003796507 & -1.0004953576 \\
$N=3$ & -0.9998209333 & -1.0001702762 & -1.0003695899 & -1.0004854553 \\
\hline
\end{tabular}

Table 7. Padé extrapolation values for the vacuum energy in Siegel gauge, from $L=4$ to $L=18$. The value of the entries where $(4 N-2=L)$ coincide with the direct $(L, 3 L)$ level-truncation computations.

\begin{tabular}{|c|c|}
\hline$L$ & $E^{\text {Sie }}$ \\
\hline 12 & -0.9998222 \\
\hline 14 & -1.0001737 \\
\hline 16 & -1.0003755 \\
\hline 18 & -1.0004937 \\
\hline
\end{tabular}

Table 8. $(L, 3 L)$ level-truncation results for the vacuum energy in Siegel gauge extracted from reference [50].

Using our known data points for the vacuum energy in Siegel gauge up to level $L=10$ which are given in table 1 , by following the same procedures shown above, we can construct the interpolating functions $f_{N}(L)$ for $N=2,3$. We have evaluated these functions at some levels, the results are presented in table 7 .

By looking at table 7, we note that the extrapolated values for the energy using either $f_{2}(L)$ or $f_{3}(L)$ for $L>10$ are almost identical. Let us remark that to construct these interpolating functions, we have only used the results up to level 10. So all values for the energy with $L>10$ are predictions that should be compared with the direct $(L, 3 L)$ level-truncation computations. For instance, in table 8, we show the results for the energy that have been obtained in reference [50] by means of direct $(L, 3 L)$ level-truncation computations.

Comparing the results for the vacuum energy given in table 7 (for $N=2$ or $N=3$ and $L>10$ ) with the results of table 8 , we conclude that the predicted values for the energy obtained by means of Padé extrapolation technique are quite well. Remarkably, the results are in agreement up to the fifth significant digit.

Analyzing these interpolating functions with $N=2$ and $N=3$, we observed that as we increase the value of the level, the function $f_{N}(L)$ decreases until reaching a minimum value $f_{N}\left(L_{\text {min }}\right)$, then for values of the level such that $L>L_{\min }$ the function $f_{N}(L)$ starts increasing and approaches asymptotically the expected value of -1 . In table 9 , we show the values of $L_{\min }$ and $f_{N}\left(L_{\min }\right)$ for $N=2,3$. This result is also in agreement with the 


\begin{tabular}{|c|c|c|}
\hline$N$ & $L_{\min }$ & $f_{N}\left(L_{\min }\right)$ \\
\hline 2 & 26.62 & -1.0006243552 \\
3 & 26.72 & -1.0006157880 \\
\hline
\end{tabular}

Table 9. Local minimum values of $f_{N}(L)$ for $N=2,3$ in Siegel gauge.

result of reference [50], where the authors have predicted that the vacuum energy will reach a minimum value close to level $L \sim 27$, and then for $L>27$ approaches asymptotically the value of -1 .

Having tested the Padé extrapolation technique, we are going to apply this method to the case of Schnabl gauge.

\subsubsection{Schnabl gauge}

To construct the interpolating functions $f_{N}(L)$, we employ the data for the vacuum energy in Schnabl gauge, this data is given in table 2 up to level $L=10$. Remember that we also need to use the asymptotic value $E^{\text {Sch }}(L \rightarrow \infty)=-1$.

As a matter of illustration, let us choose $N=2$. To determine the coefficients $a_{0}, a_{1}$, $a_{2}, b_{1}$ and $b_{2}$ that define the interpolating function of order $N=2$

$$
f_{2}(L)=\frac{a_{0}+a_{1} L+a_{2} L^{2}}{1+b_{1} L+b_{2} L^{2}}
$$

we must solve the following system of equations

$$
\begin{aligned}
f_{2}(0) & =E^{\operatorname{Sch}}(0)=-0.68461615991569, \\
f_{2}(2) & =E^{\operatorname{Sch}}(2)=-0.95937659952124, \\
f_{2}(4) & =E^{\operatorname{Sch}}(4)=-0.99465190475076, \\
f_{2}(6) & =E^{\operatorname{Sch}}(6)=-1.00398376538869, \\
f_{2}(L \rightarrow \infty) & =\frac{a_{2}}{b_{2}}=-1 .
\end{aligned}
$$

This system of equations can be easily solved to determine the corresponding values for the coefficients $a_{n}$ and $b_{n}$. Therefore, in this way we can also construct the interpolating function of order $N=3$.

We have evaluated these functions $f_{N}(L)$ at some levels, and the results are presented in table 10. Note that, as in the case of Siegel gauge, the extrapolated values for the energy using either $f_{2}(L)$ or $f_{3}(L)$ for $L>10$ are almost identical.

Analyzing the interpolating functions $f_{N}(L)$ with $N=2$ and $N=3$, we observed that as we increase the value of the level, the function $f_{N}(L)$ decreases until reaching a minimum value $f_{N}\left(L_{\text {min }}\right)$, then for values of the level such that $L>L_{\text {min }}$ the function $f_{N}(L)$ starts increasing and approaches asymptotically the expected value of -1 . In table 11 , we show the values of $L_{\min }$ and $f_{N}\left(L_{\min }\right)$ for $N=2,3$. This result is in agreement with the result obtained by means of Gaiotto-Rastelli extrapolation technique, see table 6 , where we have 


\begin{tabular}{|l|c|c|c|c|}
\hline & $L=4$ & $L=6$ & $L=8$ & $L=10$ \\
\hline$N=1$ & -0.9782901157 & -0.9851868492 & -0.9887581331 & -0.9909419314 \\
$N=2$ & & -1.0039837654 & -1.0070218437 & -1.0079880990 \\
$N=3$ & & & & -1.0081897597 \\
\hline & $L=12$ & $L=14$ & $L=16$ & $L=18$ \\
\hline$N=1$ & -0.9924153031 & -0.9934764189 & -0.9942770695 & -0.9949026734 \\
$N=2$ & -1.0081595132 & -1.0080037353 & -1.0077135987 & -1.0073747284 \\
$N=3$ & -1.0084698491 & -1.0084085581 & -1.0081970219 & -1.0079220265 \\
\hline
\end{tabular}

Table 10. Padé extrapolation values for the vacuum energy in Schnabl gauge from $L=4$ to $L=18$. The value of the entries where $(4 N-2=L)$ coincide with the direct $(L, 3 L)$ leveltruncation computations.

\begin{tabular}{|c|c|c|}
\hline$N$ & $L_{\min }$ & $f_{N}\left(L_{\min }\right)$ \\
\hline 2 & 11.76 & -1.0081618558 \\
3 & 12.43 & -1.0084765080 \\
\hline
\end{tabular}

Table 11. Local minimum values of $f_{N}(L)$ for $N=2,3$ in Schnabl gauge.

\begin{tabular}{|c|c|c|}
\hline$L$ & $E^{10}(L)$ & $f_{3}(L)$ \\
\hline 12 & -1.00847289 & -1.0084698491 \\
\hline 14 & -1.00841901 & -1.0084085581 \\
\hline 16 & -1.00821931 & -1.0081970219 \\
\hline 18 & -1.00796005 & -1.0079220265 \\
\hline
\end{tabular}

Table 12. Extrapolated values for the vacuum energy derived by means of $E^{10}(L)$ and $f_{3}(L)$, from $L=12$ to $L=18$ in Schnabl gauge.

predicted that the energy reaches a minimum value at $L \sim 12$, and then turns back to approach asymptotically -1 as $L \rightarrow \infty$.

As a matter of comparison of the two extrapolation methods studied for the case of the vacuum energy in Schnabl gauge, using $E^{10}(L)$ and $f_{3}(L)$ we have constructed table 12 , where some extrapolated values for the vacuum energy from $L=12$ to $L=18$ are shown. Note that these values can be directly extracted from tables 5 and 10 respectively. We have chosen the interpolating functions $E^{10}(L)$ and $f_{3}(L)$, because these are the best estimates we have for the vacuum energy.

By explicit $(L, 3 L)$ level-truncation computations with $L>10$, it would be interesting to confirm the above predicted results. Since the minimum value for the energy data should happen at $L \sim 12$, the direct $(L, 3 L)$ level-truncation computations must be performed, at least, up to level $L=14$.

\section{The tachyon vev}

Before analyzing the tachyon vev in the case of Schnabl gauge, we are going to study the tachyon vev in the case of Siegel gauge. 


\section{$5.1 \quad$ Siegel gauge}

In the case of Siegel gauge, the results for the tachyon vev obtained from $(L, 3 L)$ leveltruncation computations are shown in table 1 . As we can see, the value of the tachyon vev has a maximum value near level $L=4$ and then starts to decrease. We would like to know if this behavior (which we call scenario $S_{1}$ ) will continue for higher values of $L$, namely, the tachyon vev decreases for values of $L \in(4, \infty)$ and approaches monotonically to some asymptotic value as $L \rightarrow \infty$. Another possible behavior (which we call scenario $S_{2}$ ) is the one where for some very large value of $L>4$, the value of the tachyon vev stops decreasing and then starts increasing until reaching some asymptotic value as $L \rightarrow \infty$, i.e., a non-monotonic behavior. Since we do not have an analytic expression for the tachyon vacuum solution in Siegel gauge, at this point we do not know which of these two possible scenarios $S_{1}$ or $S_{2}$ will be the right one.

Scenario $S_{2}$ is compatible with the claim given in reference [61] where the analytic value $\sqrt{3} / \pi \cong 0.5513$ has been conjectured for the tachyon vev. However, in reference [50], the asymptotic value of 0.5405 has been predicted for the tachyon vev, and the authors have suggested that the conjecture [61] for an exact value $\sqrt{3} / \pi$ is falsified. Therefore, according to [50], scenario $S_{1}$ should be the correct one. Moreover, recent numerical results up to level $L=26$ seem to confirm this scenario [18]. ${ }^{4}$

Here we are going to present a criterion that will allow us to rule out one of the two scenarios. Let us start by using a rational function in $L$ to interpolate the data for the tachyon vev shown in table 1 together with the asymptotic point at $L \rightarrow \infty$

$$
R_{n, \mathrm{Sie}}^{(\alpha)}(L)=\frac{a_{0}+a_{1} L+a_{2} L^{2}+a_{3} L^{3}+\cdots+a_{n} L^{n}}{1+b_{1} L+b_{2} L^{2}+b_{3} L^{3}+\cdots+b_{n} L^{n}} .
$$

The parameter $\alpha$ has two possible values, namely, $\alpha=1$ in the case of scenario $S_{1}$ and $\alpha=2$ in the case of scneario $S_{2}$. Since we have seven data points (which include the point at infinity), we set $n=3$. The subscript Sie means that we are working in Siegel gauge.

Let us choose the asymptotic point given by the value 0.540500250625 obtained from reference [50], we require that this point together with the data of table 1 match the rational function (5.1) with $n=3$ and $\alpha=1$. Thus to determine the coefficients $a_{i}$ and $b_{i}$ we simple compare $R_{3, \text { Sie }}^{(1)}$, for each value of $L=0,2,4, \cdots, 10$ and $L \rightarrow \infty$, with all the data points. For instance, the point at infinity should be given by

$$
\lim _{L \rightarrow \infty} R_{3, \mathrm{Sie}}^{(1)}(L)=\frac{a_{3}}{b_{3}}=0.540500250625 .
$$

In this way we get a system of seven equations for the coefficients $a_{i}$ and $b_{i}$ which can be easily solved. Once these coefficients are known, the next step is to analyze the rational function $R_{3, \text { Sie }}^{(1)}(L)$.

One thing we can do is to evaluate $R_{3, \text { Sie }}^{(1)}(L)$, for values of $L>10$ and compare the results with the actual data of reference [18]. The result of these computations for values

\footnotetext{
${ }^{4}$ Actually reference [18] only discusses the results for the energy. In relation to the tachyon vev in the traditional Siegel gauge, I. Kishimoto has kindly shared with us his data of $(L, 3 L)$ level-truncation computations for levels between $L=12$ and $L=26$.
} 


\begin{tabular}{|c|c|c|c|}
\hline$L$ & $R_{3, \mathrm{Sie}}^{(1)}(L)$ & $R_{3, \mathrm{Sie}}^{(2)}(L)$ & $(L, 3 L)$ results \\
\hline 12 & 0.545607760344 & 0.545609456916 & 0.545608067009 \\
\hline 14 & 0.545074327209 & 0.545079892413 & 0.545075133495 \\
\hline 16 & 0.544635438085 & 0.544646969866 & 0.544636805350 \\
\hline 18 & 0.544270056598 & 0.544289404544 & 0.544271966369 \\
\hline 20 & 0.543962103018 & 0.543990840810 & 0.543964497784 \\
\hline 22 & 0.543699517709 & 0.543738965854 & 0.543702325407 \\
\hline 24 & 0.543473234578 & 0.543524496424 & 0.543476381413 \\
\hline 26 & 0.543276370655 & 0.543340368308 & 0.543279787348 \\
\hline
\end{tabular}

Table 13. The rational functions $R_{3, \mathrm{Sie}}^{(1)}(L)$ and $R_{3, \mathrm{Sie}}^{(2)}(L)$ compared with the direct $(L, 3 L)$ leveltruncation computations in Siegel gauge.

of $L$ between 12 to 26 are shown in table 13. Performing similar computations, but now for the case of the conjectured asymptotic value [61], namely, for scenario $S_{2}$

$$
\lim _{L \rightarrow \infty} R_{3, \mathrm{Sie}}^{(2)}(L)=\frac{a_{3}}{b_{3}}=\frac{\sqrt{3}}{\pi}=0.551328895421,
$$

we obtain another set of coefficients $a_{i}$ and $b_{i}$ which define the rational function $R_{3, \text { Sie }}^{(2)}(L)$. In table 13 , we show some values of the rational function $R_{3, \text { Sie }}^{(2)}(L)$ compared with the direct $(L, 3 L)$ level-truncation computations.

Comparing the results shown in table 13, we see that the rational interpolating function $R_{3, \text { Sie }}^{(1)}(L)$ fits better the actual data obtained by direct $(L, 3 L)$ level-truncation computations, for instance, if we compare the data starting at level $L=22$, we can observe improvement. We can take this result as a hint to choose $R_{3, \text { Sie }}^{(1)}(L)$ instead of $R_{3, \text { Sie }}^{(2)}(L)$, and thus scenario $S_{1}$ should be more likely than $S_{2}$. Next, we are going to bring another argument in favor of scenario $S_{1}$.

For a moment, let us suppose that scenario $S_{2}$ is the right one, so in this case, in order to reach the asymptotic value $\sqrt{3} / \pi \cong 0.5513$, we would like to know at which value of $L$ the tachyon vev starts to increase. From results up to level $L=26$, we can see that if there exists a point where the tachyon vev start to increase, this value must be a very high one. We can find this point using the interpolating rational function $R_{3, \text { Sie }}^{(2)}(L)$. In figure 5 , we show a plot of $R_{3, \text { Sie }}^{(2)}(L)$, and we can determine that the tachyon vev starts to increase at a point close to $L \sim 94$.

By adding the two more extra data points given in the fourth column of table 13 (the ones at $L=12$ and $L=14$ ), we can obtain the interpolating function of order $R_{4, \text { Sie }}^{(2)}(L)$. We expect that the behavior of the function $R_{4, \text { Sie }}^{(2)}(L)$ will not be much different than the function $R_{3, \text { Sie }}^{(2)}(L)$. For instance, the point where the tachyon vev starts to increase obtained by using $R_{4, \text { Sie }}^{(2)}(L)$ should be close to the one obtained by using $R_{3, \text { Sie }}^{(2)}(L)$. However, by analyzing $R_{4, \mathrm{Sie}}^{(2)}(L)$ we observe that there is no local minimum where the tachyon vev starts 


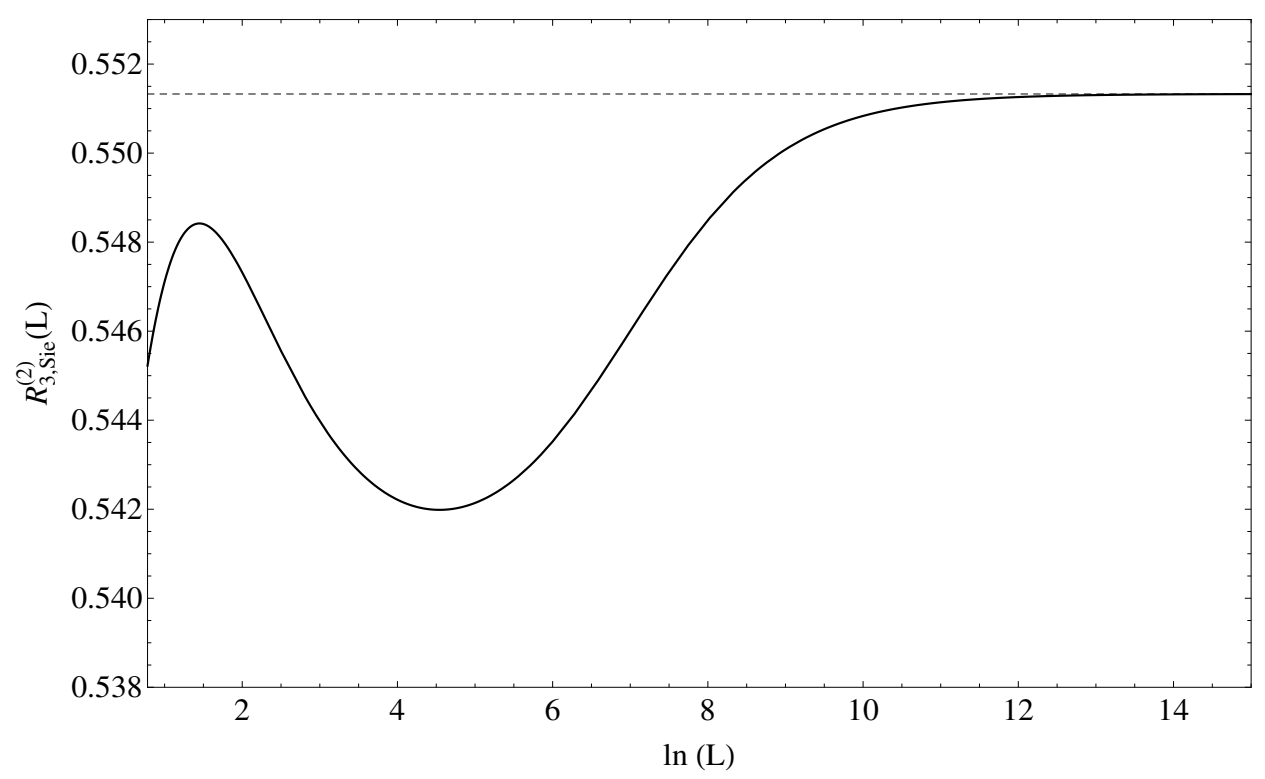

Figure 5. Plot of $R_{3, \text { Sie }}^{(2)}(L)$ for the tachyon vev as a function of level in Siegel gauge. The dashed line represents the conjectured value $\sqrt{3} / \pi$.

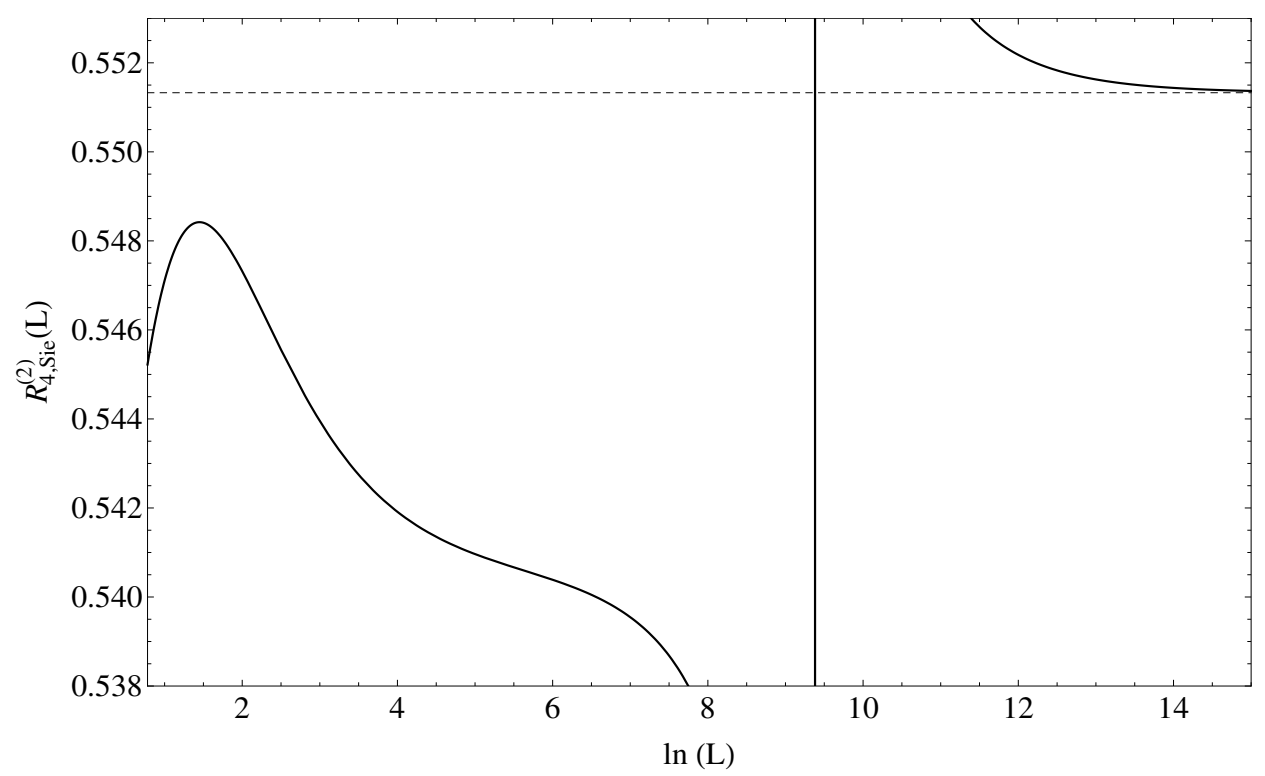

Figure 6. Plot of $R_{4, \text { Sie }}^{(2)}(L)$ for the tachyon vev as a function of level in Siegel gauge. The dashed line represents the conjectured value $\sqrt{3} / \pi$.

to increase, moreover there is a point of discontinuity near $L \sim 11908$. Figure 6 clearly illustrates our point.

From the above results and by computing higher interpolating functions (with $n>4$ ), we conclude that the set of functions $R_{n, \text { Sie }}^{(2)}(L)$ do not seem to converge to some smooth function in the limit case $n \rightarrow \infty$. This bad behavior indicates that scenario $S_{2}$ is not the correct one. 


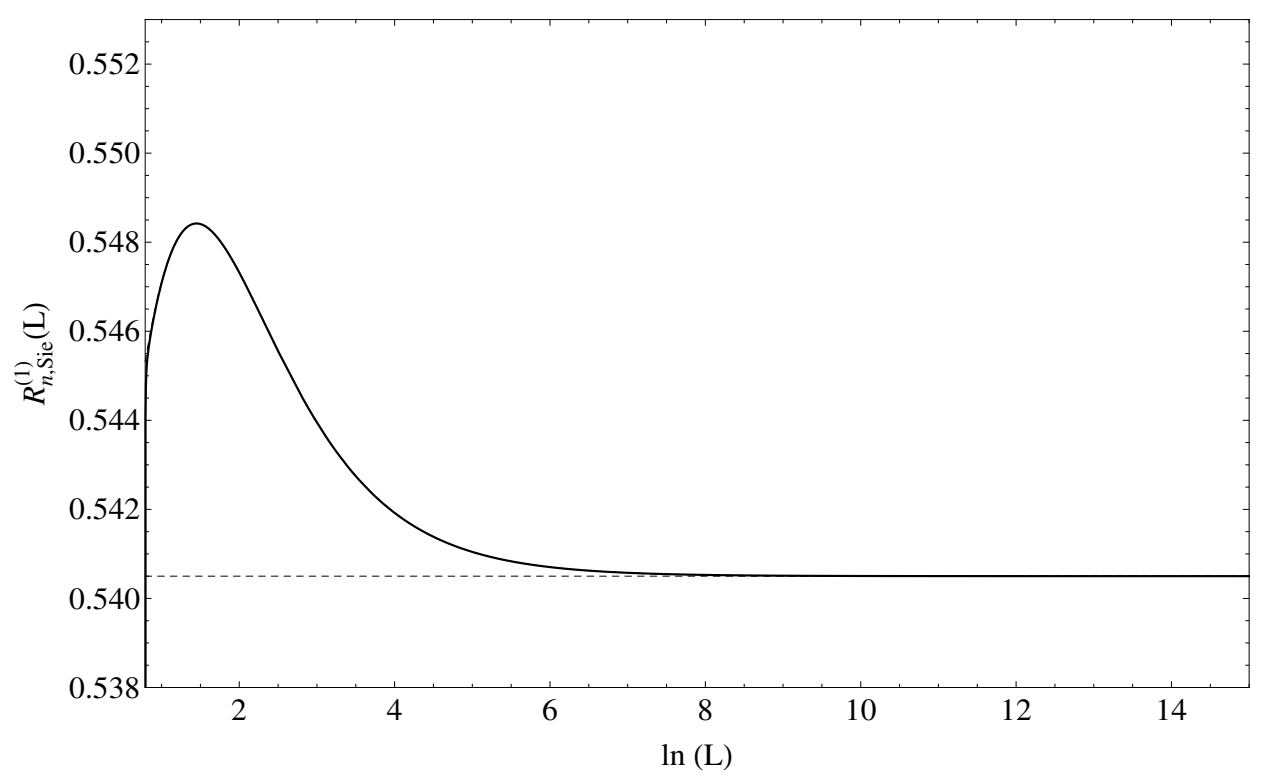

Figure 7. Plot of the functions $R_{n, \text { Sie }}^{(1)}(L)$ for the tachyon vev as a function of the level in Siegel gauge. The dashed line represents the asymptotic value 0.5405 obtained in reference [50]. The curves for $n=3,4,5,6,7$ appear superimposed in the figure.

By performing similar analysis for the case of the interpolating functions $R_{n, \mathrm{Sie}}^{(1)}(L)$, namely, for scenario $S_{1}$ we get a nice behavior that is illustrated in figure 7 , the functions $R_{n, \text { Sie }}^{(1)}(L)$ converge to a smooth function in the limit case when $n \rightarrow \infty$. This is a clear indication that scenario $S_{1}$ is the right one. In the following subsection, we are going to analyze the case of Schnabl gauge.

\subsection{Schnabl gauge}

The results for the tachyon vev in Schnabl gauge obtained from $(L, 3 L)$ level-truncation computations are shown in table 2. Note that as in the case of Siegel gauge, the value of the tachyon vev has a maximum value near level $L=4$ and then starts to decrease.

Since in the case of Schnabl gauge the analytic (asymptotic) value of the tachyon vev is known [1], the two scenarios $S_{1}$ and $S_{2}$ should be the same. Let us compute the interpolating functions $R_{n, \text { Sch }}^{(2)}(L)$ corresponding to scenario $S_{2}$.

We use a rational function in $L$ to interpolate the data for the tachyon vev shown in table 2 together with the asymptotic point at $L \rightarrow \infty$

$$
R_{3, \text { Sch }}^{(2)}(L)=\frac{a_{0}+a_{1} L+a_{2} L^{2}+a_{3} L^{3}}{1+b_{1} L+b_{2} L^{2}+b_{3} L^{3}} .
$$

Since we have seven data points (which include the point at infinity), we have set $n=3$. As usual, to obtain the seven unknown coefficients $a_{i}$ and $b_{i}$, we require that the data points in table 2 coincide with the rational function (5.4) evaluated at the known values of the direct $(L, 3 L)$ level-truncation computations for $L=0,2,4,6,7,10$ and the asymptotic one $L \rightarrow \infty$ (the analytic result). For instance, at level $L=0$, we obtain

$$
R_{3, \mathrm{Sch}}^{(2)}(L=0)=a_{0}=0.456177990470,
$$




\begin{tabular}{|c|c|}
\hline$L$ & $R_{3, \mathrm{Sch}}^{(2)}(L)$ \\
\hline 12 & 0.545904273712 \\
\hline 14 & 0.545468779861 \\
\hline 16 & 0.545160525135 \\
\hline 18 & 0.544947247026 \\
\hline 20 & 0.544805165981 \\
\hline 22 & 0.544716903893 \\
\hline 24 & 0.544669683450 \\
\hline 26 & 0.544654007673 \\
\hline
\end{tabular}

Table 14. The rational function $R_{3, \mathrm{Sch}}^{(2)}(L)$ for some values of $L>10$.

while using the asymptotic value, we have the following equation

$$
\lim _{L \rightarrow \infty} R_{3}^{(2)}(L)=\frac{a_{3}}{b_{3}}=0.553465566934
$$

In this way, we get a system of seven equations for the coefficients $a_{i}$ and $b_{i}$ which can be easily solved. Once these coefficients are known, the next step is to analyze the rational function $R_{3, \text { Sch }}^{(2)}(L)$.

Let us evaluate $R_{3, \text { Sch }}^{(2)}(L)$ for values of $L>10$ between 12 to 26, the results are shown in table 14. Note that for levels close to $L=24$, the value of the tachyon vev seems to stop decreasing. At levels, between $L=24$ and $L=26$ the value of the tachyon vev is almost the same, this indicates that we are close to a point where we have reached a local minimum. In figure 8, we show the plot of $R_{3, \mathrm{Sch}}^{(2)}(L)$, as we can see, at level close to $L=26$, the tachyon vev starts increasing and then approaches asymptotically the analytic value shown as the dashed line.

By adding two more extra data points obtained by direct $(L, 3 L)$ level-truncation computations for $L=12$ and $L=14$, we can derive the interpolating function of order $R_{4, \mathrm{Sch}}^{(2)}(L)$. We expect that the behavior of this function $R_{4, \mathrm{Sch}}^{(2)}(L)$ will not be much different from the function $R_{3, \mathrm{Sch}}^{(2)}(L)$, for instance, the point where the tachyon vev starts to increase obtained by using $R_{4, \text { Sch }}^{(2)}(L)$ should be close to the one obtained by using $R_{3, \text { Sch }}^{(2)}(L)$. In fact, this behavior is observed as shown in figure 9 . We expect that when $n \rightarrow \infty$ the set of functions $R_{n, \text { Sch }}^{(2)}(L)$ converges to some smooth function.

To be honest, since we have not computed the values of the tachyon vev for $L=12$ and $L=14$ by means of direct $(L, 3 L)$ level-truncation computations, to derive the interpolating function $R_{4, \mathrm{Sch}}^{(2)}(L)$ we have used the data shown in the second column of table 14 for $L=12$ and $L=14$ with four digits of precision, namely, the values 0.5459 and 0.5454 respectively. We hope that the actual $(L, 3 L)$ level-truncation computation will confirm these values for the tachyon vev. 


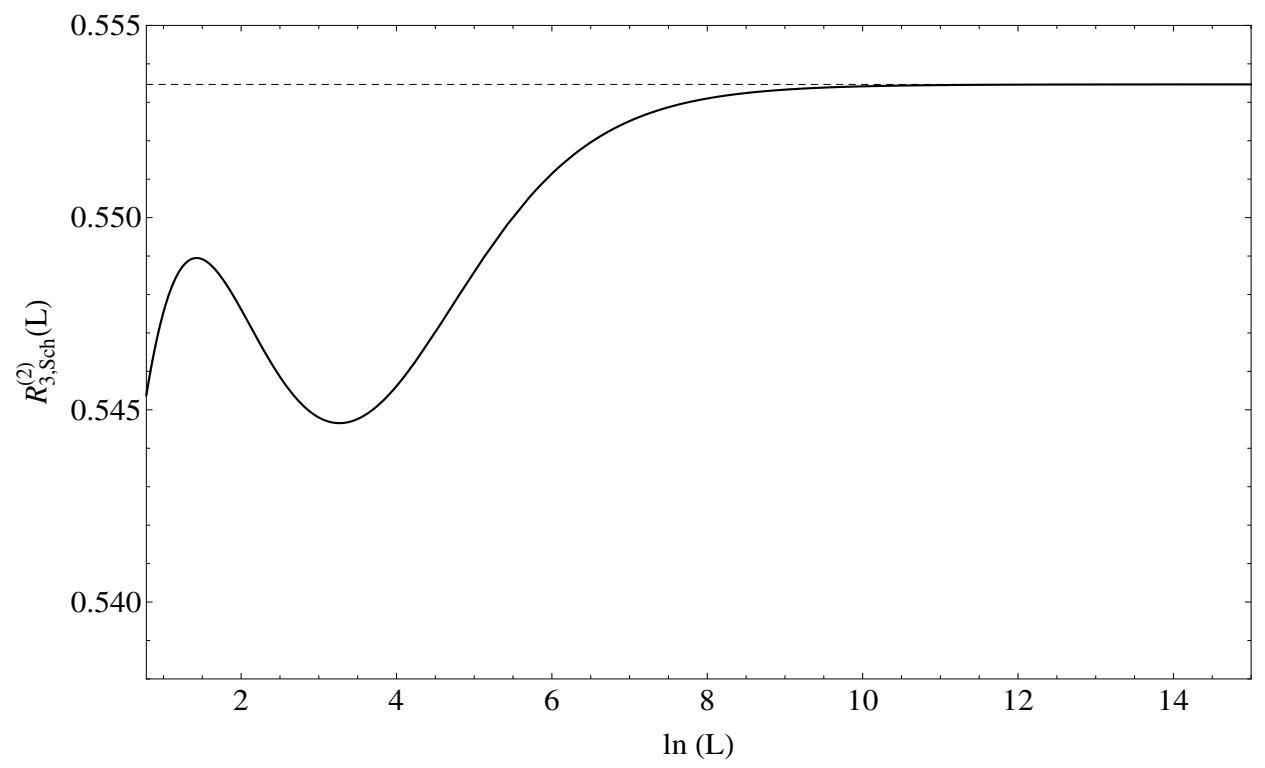

Figure 8. Plot of the function $R_{3, \mathrm{Sch}}^{(2)}(L)$ shown as the continuous line. The dashed line represents the asymptotic value 0.5534 obtained in reference [1].

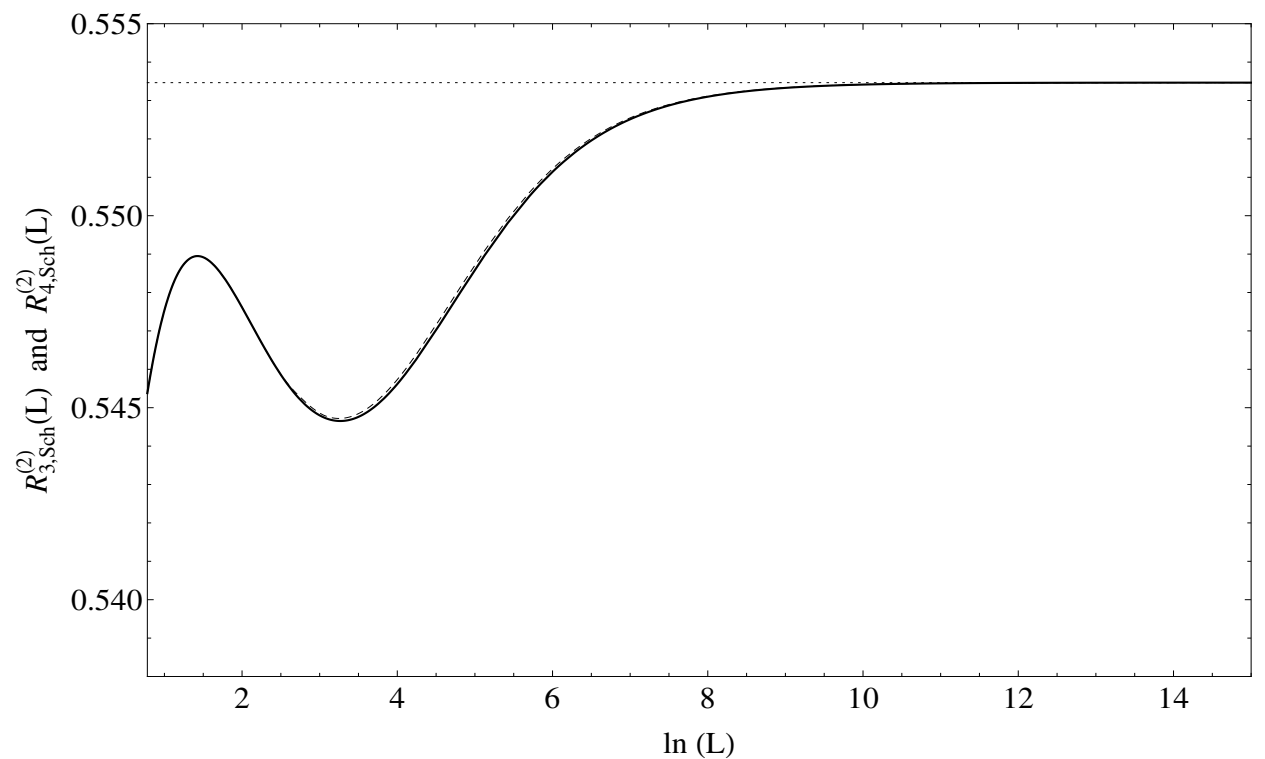

Figure 9. Plot of the function $R_{3, \mathrm{Sch}}^{(2)}(L)$ shown as the continuous line compared with the function $R_{4, \mathrm{Sch}}^{(2)}(L)$ represented as the dashed line for the tachyon vev as a function of the level in Schnabl gauge. The dotted line represents the asymptotic value 0.5534 obtained in reference [1].

The above results suggest that at level close to $L \sim 26$, the value of the tachyon vev starts to increase until reaching the asymptotic value of 0.5534 . Figure 9 illustrates this point. We leave as a future research project, the test of the validity of this prediction by means of direct $(L, 3 L)$ level-truncation computations for levels $L>10$. 


\section{Summary and discussion}

Using either Siegel or Schnabl gauge, we have constructed the effective tachyon potential and analyzed its branch structure by means of Virasoro $L_{0}$ level-truncation computations. It would be interesting to extend this analysis to higher levels and to probe the physical branch in a much larger region. We should use a different gauge such that its region of validity must be greater than Siegel or Schnabl gauges, for instance, we could explore the so-called linear $b$-gauges studied in reference [16].

Selecting the physical branch of the effective tachyon potential in Schnabl gauge, namely, the branch that connects the perturbative with non-perturbative vacuum and computing its local minimum, we have derived data points for the energy as well as for the tachyon vev.

Regarding the data for the energy obtained by direct $(L, 3 L)$ level-truncation computations, we have observed that at level $L=6$ the energy overshoots the expected analytical answer of -1 , and appears to further decrease at higher levels. This result indicates that the approach of the energy to -1 as $L \rightarrow \infty$ is non-monotonic. By applying two kind of extrapolation techniques to the level-truncation data for $L \leq 10$ to estimate the vacuum energies even for $L>10$, we have predicted that the energy reaches a minimum value at $L \sim 12$, and then turns back to approach asymptotically -1 as $L \rightarrow \infty$.

Regarding the data for the tachyon vev, we have found that starting at level $L=4$, the value of the tachyon vev decreases. To reach the analytical value of 0.553465 , we have noted that there should be some higher value of $L>4$ such that the value of the tachyon vev stops decreasing and then starts increasing until reaching this analytical value. We have predicted that for $L>4$ the tachyon vev reaches a minimum value for $L \sim 26$, and then turns back to approach asymptotically the expected analytical result.

By explicit $(L, 3 L)$ level-truncation computations with $L>10$, it would be interesting to confirm the above predicted results. Since the minimum value of the energy data should happen at $L \sim 12$, the direct $(L, 3 L)$ level-truncation computations must be performed, at least, up to level $L=14$. While in the case of the tachyon vev data, to confirm the existence of a minimum value close to level $L \sim 26$, we will need to perform the calculations, at least, up to level $L=28$. These issues will be the subject of a future research project.

Finally, since the modified cubic superstring field theory [62] as well as Berkovits superstring field theory [63] are based on Witten's associative star product of open bosonic string field theory, using rather generic gauge conditions, our results can be naturally extended to analyze numerical solutions in the context of these open superstring field theories.

\section{Acknowledgments}

EAA would like to thank Ted Erler and Isao Kishimoto for useful discussions. The research of AFS is supported by CAPES grant 1675676. The research of RS is supported by CAPES grant 1627292 . 
Open Access. This article is distributed under the terms of the Creative Commons Attribution License (CC-BY 4.0), which permits any use, distribution and reproduction in any medium, provided the original author(s) and source are credited.

\section{References}

[1] M. Schnabl, Analytic solution for tachyon condensation in open string field theory, Adv. Theor. Math. Phys. 10 (2006) 433 [hep-th/0511286] [INSPIRE].

[2] E. Witten, Noncommutative geometry and string field theory, Nucl. Phys. B 268 (1986) 253 [INSPIRE].

[3] A. Sen, Descent relations among bosonic D-branes, Int. J. Mod. Phys. A 14 (1999) 4061 [hep-th/9902105] [INSPIRE].

[4] A. Sen, Universality of the tachyon potential, JHEP 12 (1999) 027 [hep-th/9911116] [INSPIRE].

[5] Y. Okawa, Comments on Schnabl's analytic solution for tachyon condensation in Witten's open string field theory, JHEP 04 (2006) 055 [hep-th/0603159] [INSPIRE].

[6] Y. Okawa, Analytic methods in open string field theory, Prog. Theor. Phys. 128 (2012) 1001 [INSPIRE].

[7] E. Fuchs and M. Kroyter, Schnabl's L(0) operator in the continuous basis, JHEP 10 (2006) 067 [hep-th/0605254] [INSPIRE].

[8] E.A. Arroyo, Conservation laws and tachyon potentials in the sliver frame, JHEP 06 (2011) 033 [arXiv: 1103.4830] [INSPIRE].

[9] L. Rastelli and B. Zwiebach, Solving open string field theory with special projectors, JHEP 01 (2008) 020 [hep-th/0606131] [INSPIRE].

[10] I. Ellwood and M. Schnabl, Proof of vanishing cohomology at the tachyon vacuum, JHEP 02 (2007) 096 [hep-th/0606142] [INSPIRE].

[11] Y. Okawa, L. Rastelli and B. Zwiebach, Analytic solutions for tachyon condensation with general projectors, hep-th/0611110 [INSPIRE].

[12] T. Erler, Split string formalism and the closed string vacuum, JHEP 05 (2007) 083 [hep-th/0611200] [INSPIRE].

[13] T. Erler, Split string formalism and the closed string vacuum. II, JHEP 05 (2007) 084 [hep-th/0612050] [INSPIRE].

[14] M. Schnabl, Algebraic solutions in open string field theory - A lightning review, arXiv: 1004.4858 [INSPIRE].

[15] E. Fuchs and M. Kroyter, Analytical solutions of open string field theory, Phys. Rept. 502 (2011) 89 [arXiv:0807.4722] [INSPIRE].

[16] M. Kiermaier, A. Sen and B. Zwiebach, Linear b-gauges for open string fields, JHEP 03 (2008) 050 [arXiv:0712.0627] [INSPIRE].

[17] T. Takahashi, Level truncation analysis of exact solutions in open string field theory, JHEP 01 (2008) 001 [arXiv:0710.5358] [INSPIRE].

[18] I. Kishimoto, On numerical solutions in open string field theory, Prog. Theor. Phys. Suppl. 188 (2011) 155 [INSPIRE]. 
[19] E.A. Arroyo, Cubic interaction term for Schnabl's solution using Pade approximants, J. Phys. A 42 (2009) 375402 [arXiv:0905.2014] [InSPIRE].

[20] E. Aldo Arroyo, Level truncation analysis of regularized identity based solutions, JHEP 11 (2011) 079 [arXiv:1109.5354] [INSPIRE].

[21] E.A. Arroyo, Comments on regularization of identity based solutions in string field theory, JHEP 11 (2010) 135 [arXiv:1009.0198] [INSPIRE].

[22] T. Takahashi and S. Tanimoto, Marginal and scalar solutions in cubic open string field theory, JHEP 03 (2002) 033 [hep-th/0202133] [INSPIRE].

[23] T. Erler and M. Schnabl, A simple analytic solution for tachyon condensation, JHEP 10 (2009) 066 [arXiv:0906.0979] [INSPIRE].

[24] M. Murata and M. Schnabl, Multibrane solutions in open string field theory, JHEP 07 (2012) 063 [arXiv:1112.0591] [INSPIRE].

[25] T. Masuda, Comments on new multiple-brane solutions based on Hata-Kojita duality in open string field theory, JHEP 05 (2014) 021 [arXiv: 1211.2649] [INSPIRE].

[26] H. Hata and T. Kojita, Singularities in K-space and multi-brane solutions in cubic string field theory, JHEP 02 (2013) 065 [arXiv: 1209.4406] [INSPIRE].

[27] T. Masuda, T. Noumi and D. Takahashi, Constraints on a class of classical solutions in open string field theory, JHEP 10 (2012) 113 [arXiv: 1207.6220] [INSPIRE].

[28] L. Bonora, S. Giaccari and D.D. Tolla, Analytic solutions for Dp-branes in SFT, JHEP 12 (2011) 033 [arXiv:1106.3914] [INSPIRE].

[29] L. Bonora, S. Giaccari and D.D. Tolla, The energy of the analytic lump solution in SFT, JHEP 08 (2011) 158 [Erratum ibid. 04 (2012) 001] [arXiv:1105.5926] [INSPIRE].

[30] M. Kiermaier, Y. Okawa, L. Rastelli and B. Zwiebach, Analytic solutions for marginal deformations in open string field theory, JHEP 01 (2008) 028 [hep-th/0701249] [INSPIRE].

[31] N. Mertes and M. Schnabl, String field representation of the Virasoro algebra, JHEP 12 (2016) 151 [arXiv:1610.00968] [INSPIRE].

[32] M. Jokel, Real tachyon vacuum solution without square roots, arXiv:1704.02391 [INSPIRE].

[33] E.A. Arroyo, Comments on real tachyon vacuum solution without square roots, arXiv: 1706.00336 [INSPIRE].

[34] Y. Okawa, Analytic solutions for marginal deformations in open superstring field theory, JHEP 09 (2007) 084 [arXiv:0704.0936] [INSPIRE].

[35] T. Erler, Tachyon vacuum in cubic superstring field theory, JHEP 01 (2008) 013 [arXiv:0707.4591] [INSPIRE].

[36] E.A. Arroyo, Generating Erler-Schnabl-type solution for tachyon vacuum in cubic superstring field theory, J. Phys. A 43 (2010) 445403 [arXiv:1004.3030] [InSPIRE].

[37] R.V. Gorbachev, New solution of the superstring equation of motion, Theor. Math. Phys. 162 (2010) 90 [Teor. Mat. Fiz. 162 (2010) 106] [INSPIRE].

[38] I.Ya. Aref'eva, R.V. Gorbachev and P.B. Medvedev, Pure gauge configurations and solutions to fermionic superstring field theories equations of motion, J. Phys. A 42 (2009) 304001 [arXiv: 0903.1273] [INSPIRE]. 
[39] E.A. Arroyo, A singular one-parameter family of solutions in cubic superstring field theory, JHEP 05 (2016) 013 [arXiv: 1602.00059] [INSPIRE].

[40] E. Aldo Arroyo, Level truncation analysis of a simple tachyon vacuum solution in cubic superstring field theory, JHEP 12 (2014) 069 [arXiv:1409.1890] [INSPIRE].

[41] E. Aldo Arroyo, Comments on multibrane solutions in cubic superstring field theory, PTEP 2014 (2014) 063B03 [arXiv: 1306.1865] [INSPIRE].

[42] E. Aldo Arroyo, Multibrane solutions in cubic superstring field theory, JHEP 06 (2012) 157 [arXiv: 1204.0213] [INSPIRE].

[43] T. Erler, Exotic universal solutions in cubic superstring field theory, JHEP 04 (2011) 107 [arXiv: 1009.1865] [INSPIRE].

[44] T. Erler, Marginal solutions for the superstring, JHEP 07 (2007) 050 [arXiv:0704.0930] [INSPIRE].

[45] T. Erler, Analytic solution for tachyon condensation in Berkovits' open superstring field theory, JHEP 11 (2013) 007 [arXiv: 1308.4400] [INSPIRE].

[46] V.A. Kostelecky and S. Samuel, On a nonperturbative vacuum for the open bosonic string, Nucl. Phys. B 336 (1990) 263 [inSPIRE].

[47] A. Sen and B. Zwiebach, Tachyon condensation in string field theory, JHEP 03 (2000) 002 [hep-th/9912249] [INSPIRE].

[48] L. Rastelli and B. Zwiebach, Tachyon potentials, star products and universality, JHEP 09 (2001) 038 [hep-th/0006240] [inSPIRE].

[49] N. Moeller and W. Taylor, Level truncation and the tachyon in open bosonic string field theory, Nucl. Phys. B 583 (2000) 105 [hep-th/0002237] [INSPIRE].

[50] D. Gaiotto and L. Rastelli, Experimental string field theory, JHEP 08 (2003) 048 [hep-th/0211012] [INSPIRE].

[51] W. Taylor, A perturbative analysis of tachyon condensation, JHEP 03 (2003) 029 [hep-th/0208149] [INSPIRE].

[52] M. Asano and M. Kato, Level truncated tachyon potential in various gauges, JHEP 01 (2007) 028 [hep-th/0611190] [INSPIRE].

[53] I. Kishimoto and T. Takahashi, Numerical evaluation of gauge invariants for a-gauge solutions in open string field theory, Prog. Theor. Phys. 121 (2009) 695 [arXiv:0902.0445] [INSPIRE].

[54] I. Kishimoto and T. Takahashi, Numerical evaluation of gauge invariants for a-gauge solutions in open string field theory, Theor. Math. Phys. 163 (2010) 710 [arXiv:0910.3025] [INSPIRE].

[55] E. Aldo Arroyo, The tachyon potential in the sliver frame, JHEP 10 (2009) 056 [arXiv: 0907.4939] [INSPIRE].

[56] M.R. Gaberdiel and B. Zwiebach, Tensor constructions of open string theories. 1: Foundations, Nucl. Phys. B 505 (1997) 569 [hep-th/9705038] [INSPIRE].

[57] A.A. Gerasimov and S.L. Shatashvili, On exact tachyon potential in open string field theory, JHEP 10 (2000) 034 [hep-th/0009103] [INSPIRE]. 
[58] D. Kutasov, M. Mariño and G.W. Moore, Some exact results on tachyon condensation in string field theory, JHEP 10 (2000) 045 [hep-th/0009148] [INSPIRE].

[59] D. Ghoshal and A. Sen, Normalization of the background independent open string field theory action, JHEP 11 (2000) 021 [hep-th/0009191] [INSPIRE].

[60] I. Ellwood and W. Taylor, Gauge invariance and tachyon condensation in open string field theory, hep-th/0105156 [INSPIRE].

[61] H. Hata and S. Shinohara, BRST invariance of the nonperturbative vacuum in bosonic open string field theory, JHEP 09 (2000) 035 [hep-th/0009105] [INSPIRE].

[62] I.Ya. Arefeva, P.B. Medvedev and A.P. Zubarev, New representation for string field solves the consistency problem for open superstring field theory, Nucl. Phys. B 341 (1990) 464 [INSPIRE].

[63] N. Berkovits, Super-Poincaré invariant superstring field theory, Nucl. Phys. B 450 (1995) 90 [Erratum ibid. B 459 (1996) 439] [hep-th/9503099] [INSPIRE]. 